\title{
Chemical entropy generation and second- order slip condition on hydrodynamic Casson nanofluid flow embedded in a porous medium: a fast convergent method
}

\author{
Adebowale Martins Obalalu ${ }^{1,2^{*}}$
}

${ }^{*}$ Correspondence:

adebowale.

obalalu17@kwasu.edu.ng

1 Department of Physics,

Augustine University llara

Epe, Lagos, Nigeria

Full list of author information

is available at the end of the

article

\begin{abstract}
The chemical entropy generation analysis is an approach to optimize the performance of different thermal systems by investigating the related irreversibility of the system. The influences of second-order slip with the chemical reaction on the boundary layer flow and heat transfer of a non-Newtonian nanofluid in a non-Darcian porous medium have been investigated numerically. Simultaneous solutions are presented for first and second-order velocity slips. The second-order boundary conditions serve as a closure of a system of the continuity, transport, and energy differential equations. The current work differs from the previous studies in the application of a new second-order slip velocity model. The Casson fluid model is applied to characterize the non-Newtonian fluid behavior. The effect of the second slip parameter on the present physical parameters was discussed through graphs and it was found that this type of slip is a very important one to predict the investigated physical model. The present study provides two fast convergent methods on the semi-infinite interval, namely Chebyshev collocation method and optimal homotopy analysis method are used to analyze the fluid flow, heat, and mass transport. Compared with available analytical and numerical solutions, current methods are effective, quickly converging, and with great accuracy. It was shown that the account for the second-order terms in the boundary conditions noticeably affects the fluid flow characteristics and does not influence on the heat transfer characteristics.
\end{abstract}

Keywords: Casson nanofluid, Chemical reaction, Chebyshev collocation method, Optimal homotopy analysis method

Mathematics Subject Classification: 74A15, 76A05, 34B40, 65B10

\section{Introduction}

In recent years, there has been an increasing interest in the optimization of chemical processes, leading to Energy savings or enhancing their efficiency [1]. A chemical reaction is a reversible or irreversible process in which two different types of chemical

(c) The Author(s) 2022. Open Access This article is licensed under a Creative Commons Attribution 4.0 International License, which permits use, sharing, adaptation, distribution and reproduction in any medium or format, as long as you give appropriate credit to the original author(s) and the source, provide a link to the Creative Commons licence, and indicate if changes were made. The images or other third party material in this article are included in the article's Creative Commons licence, unless indicated otherwise in a credit line to the material. If material is not included in the article's Creative Commons licence and your intended use is not permitted by statutory regulation or exceeds the permitted use, you will need to obtain permission directly from the copyright holder. To view a copy of this licence, visit http:// creativecommons.org/licenses/by/4.0/. 
constituents react to form a single product in the presence of an enzyme or catalyst. Chemical reactions are extremely important to process cheap raw materials into highvalue products in the chemical processing industries [2]. This phenomenon plays a significant role in the chemical industry, power, and cooling industry for drying, evaporation, energy transfer in a cooling tower, and the flow in a desert cooler, etc. The study of heat transfer with chemical reaction is of most realistic significance to engineers and scientists because of its universal incidence in many branches of science and engineering. Activation energy is the smallest amount of energy that must be required to operate molecules or atoms in a chemical system that can start a chemical reaction. The activation energy was also first proposed by Svante Arrhenius in 1899 [3]. Activation energy is considered as the separating barrier between two energy states. A chemical reaction will start whenever this energy is crossed. At a reasonable rate to proceed with a chemical reaction, there exist a significant number of atoms or molecules with translational energy treater than or equal to the activation energy. Its applications can be found in oil repository designing, geothermal, and mechanics of water [4]. The effect of Arrhenius energy and chemical reaction on hydromagnetic nanofluid of Casson flow of two dimensional electrically conducting thermal were numerical study by $[5,6]$.

In thermodynamics, the measure of disorder is called entropy. According to the second law of thermodynamics for an isolated system, the system spontaneously grows toward thermodynamic equilibrium and attains minimum entropy. On the other hand, for a non-isolated system, there is a possibility of a decrease in entropy of a system, which may transfer the same amount of entropy to surroundings. Heat transfer and viscous dissipation play vital roles in changing the behavior of entropy of a system. The study of entropy generation is a field of interest among researchers. Entropy generation was highlighted as an important instrument for improving the efficiency of the heat transfer operation. The design of new thermal systems is more important to industries developing heat transfer technology; therefore, several researchers have attempted to investigate the entropy generation and heat transfer performance of novel materials of heat transfer fluid. Obalalu et.al [7] recently used entropy generation to assess the importance of irreversibility in heat transfer, friction, and other nonideal processes within thermal systems. The impact of heat transfer together with entropy generation has been used intensively since the pioneering work by Bejan which involves the study of thermodynamics process within the boundary-layer flow [8]. The new generation of entropy and stagnant point flows have been studied by [9]. A computational analysis of Casson flow Nanofluid flow in a stretching surface of entropy generation [10]. It was observed that the non-Newtonian Casson parameter has been shown to improve the generation entropy number. A novel matrix technique for multi-order pantograph differential equations of fractional order have been examined by [11]. Some dynamical models involving fractional-order derivatives with the Mittag-Leffler type kernels and their applications based upon the Legendre spectral collocation method have been examined by [12]. A discretization approach for the nonlinear fractional logistic equation were investigated by [13]. The numerical approximations to the nonlinear fractional-order Logistic population model with fractional-order Bessel and Legendre bases were investigated by [14].

Meanwhile, since the pioneering work [15] studies related to the magnetohydrodynamics (MHD) is One of the important areas of development in modern scientific 
research and engineering problems is magnetohydrodynamics this scientific field can also be considered as a fluid mechanics subdiscipline dealing with the mutual interactions between an externally imposed magnetic field and the flows of fluids that conduct electricity [7]. The study of magnetic properties and compliance of electrically conducting liquids is magnetic hydrodynamics. Examples include plasmas, molten metals, saltwater, and electrolytes. Electrically conducting heat and mass carrier fluids which include water, mineral oil, ethylene glycol, and so on are found useful in industry and engineering for industrial coolant, brake fluid, car radiator coolant, MHD generator, electronic voice coil cooling, nano-drug delivery, cancer therapy[16, 17]. A comprehensive review of the literature about the effect of a chemical reaction on magnetohydrodynamic (MHD) is given by references [18-20]. In science, the electrical conduction of heat and heat transfer fluid, including water, mineral oil, and ethylene glycol is significant because it is used in manufacturing, engineering, industrial coolers, brake fluid, and automobile radiator coolant, However, these fluids have low thermophysical properties that impede their usage in some places [21]. By adding ultrasound nanoparticles usually made from metal or metallic oxides to such fluids, the thermophysical properties of those fluids can be enhanced. The nanofluid can be used for various applications using a magnetic field. This includes the care of cuts, gastric medicine, sterilized instruments, etc. [22]. In the case of magnetic resonance imaging (MRI), high thermal hyperthermia, magnetic drug targeting, and tissue engineering, bio-suspension based upon the magnetic nanoparticle [23]. The work of $\mathrm{Yu}$ and Xie [24] contributes greatly to the recent developments of nanofluids preparations and upcoming applications in several fields. They suggested methods for improving the stability of nanofluids. The Numerical study of higher-order chemical reactions to MHD nanofluid electrically influenced by viscous dissipation was investigated by [25].

In many branches of many fields of science, engineering, and food processing, Casson fluid (CF) has received significant attention Oil, honey, jelly, and paint are common examples of common commodities exhibiting CF properties [26]. Casson fluid (CF) is a non-Newtonian fluid with shear thinning, yield stress, and high shear viscosity. It acts like an elastic solid at low shear strain, while it acts like a Newtonian fluid at above critical yield stress [27] Casson first established the CF model [28]. The study defines the pigment-oil suspension prediction flow. The Newtonian fluid model was shown to decrease to non-Newtonian fluid particularly when the stress on the wall is above the yield stress [29]. CF flow from hydromagnetic and thermal convection heat transfer to a stretched permeable surface was reported [30]. Navier's slip condition was incorporated in the above-reviewed literature. This condition is good enough (Navier slip) at low shear rates. The slip condition of Navier however decreases as the slip length increases quickly with the slip rate [31]. The second-order slip boundary condition is therefore considered (a non-Navier slip). The rarefaction and compression effects of gas microflows have been studied by [32]. The results obtained were compared with test data. They observed that the calculated values are closer to the experimental values by using the second-order slip condition.

The transformed ordinary differential equation (ODE) mostly with related boundary conditions becomes a strongly non-linear boundary value problem (BVP) method. A differential equation is an ordinary differential equation (ODE) that contains one or 
more functions and the derivatives of one independent variable. A boundary value problem is a differential equation combined with several extra conditions called boundary conditions in the field of differential equations [33]. The solution of these non-linear (BVP) continues to fascinate and motivate scientists to create methods for obtaining solutions that explain the complex characteristics of the underlying problem of boundary layer flow under various conditions. The numerical approach is the standard way to solve the transformed similarity variable boundary layer equations, Numerical analyzes are needed to overcome engineering problems leading to equations that cannot be analytically resolved by simple formulas. Examples of such problems occur from natural sciences, social sciences, engineering, medicine, and industries in major systems of algebraic equations, integral evaluations, and the solution of differential equations [34]. The numerical methods that some researchers have chosen include the collocation method [35]. The weighted residual method [36, 37], finite difference method [38], finite different element [39], Runge-Kutta scheme [40], different kinds of spectral methods for solving problems in bounded domains or under particular boundary conditions have been explored in many studies some analytical approaches have been found very useful In the analysis of magneto-hydrodynamic Casson nanofluid flow, Analytic solution in cases of severe nonlinearity is important. The application of analytical methods is restrictive and can be used only in a few equations. however, most analytical methods in fluid mechanics relating to physical applications, it is illogical to utilize the analytical technique, since their solution turns out to be too cumbersome and it can be very slow or not possible to converge on the real solution. For this explanation, the numerical method is the most realistic way of finding a solution for extremely nonlinear structures of boundary layer flow. Techniques of computational numerical solutions likewise have their difficulty, including the problem of stability and convergence. Several studies in this regard have been carried out following innovative research. Liao [41] proposed the Homotopy Analysis Method (HAM) that is a powerful analytical technique, providing nonlinear differential equations with power series solutions. There are no small or large parameters in this technique, as is customary in conventional perturbation. By using perturbation methods identified analytical solutions to weak nonlinear boundary value problems, but this method cannot be used for certain specified ranges of parameters in many problems [42]. In the presence of heat generation [43] used a homotopy disturbance method for the effect of variable thermal conductivity on heat transfer through the hollow sphere. For the nonlinear solution, other analytical approaches were used Differential equations method of Lindstedt-Poincare method of linearization, optimal method of homotopy perturbation, and method of differential transformation. An efficient semi-analytical method for solving the generalized regularized long wave equations with a new fractional derivative operator have been investigated by [44]. The oscillatory states and patterns formation in a model subjected to the dirichlet conditions have been examined by [45]. The computational Method for reaction diffusion-model arising in a spherical catalyst were investigated by [46]. A reliable numerical algorithm for fractional advection-dispersion equation arising in contaminant transport through porous media [47]. Jacobi collocation method for the fractional advection-dispersion equation arising in porous media was investigated by [48]. Chebyshev spectral method for solving a class of local and nonlocal elliptic boundary value problems [49]. An introductory overview of 
fractional-calculus operators based upon the Fox-Wright and related higher transcendental functions have been examined by [50].

Many methods for heat and mass transfer study of hydromagnetic Casson nanofluid flow have been used, according to literature reviews. The terms chemical reaction, Arrhenius activation, and second-order (non-Navier) velocity slip has not been considered using numerical and analytical approaches namely Chebyshev Collocation Method (CCM) and Optimal Homotopy Analysis Method (OHAM) until now. The second law of thermodynamic in this study was extended to the analysis of the fluid flow, heat, and mass transfer and the dimensions of entropy generation with a rheological Casson model. The present work essentially extends the recent work of Titiloye et al. [51] to include the second law of thermodynamic on hydromagnetic non-Darcian Casson nanofluid. Due to its wide applications in food processing, paper and textile dying, bio-liquid flow, drug production, and pharmaceuticals, the Casson rheological model is considered in this study. The parameter is included in the flow sector, due to their great contribution to the various physical dimensionless quantities as mentioned in the above paragraphs and to better predict the flow. The heat and mass transfer are computed using the Chebyshev collocation method (CCM). This Scheme involves: assuming Chebyshev base form solutions with unknown coefficients for the unknown dependent functions in the differential equations; The assumed solutions are substituted into the governing equations to produce residuals or errors; the errors are then forced to become zero using collocation method; A system of algebraic equations is derived and then solved to obtain the values of the unknown coefficients. The obtained results are computed and discussed using tables and graphs. The concluding remarks are also presented. The method form is novel and can be used to build a model for blood oxygenators and hemodialyzers.

\section{Mathematical model}

Consider a non-Darcian porous medium with a two-dimensional incompressible MHD chemically reactive Casson nanofluid flow over a stretching sheet (see Fig. 1). $\bar{x}$ denotes the distance along the line, while $\bar{y}$ denotes the distance perpendicular to the sheet. The fluid's properties are believed to be constant during the simulation. $T$ and $C$ represent

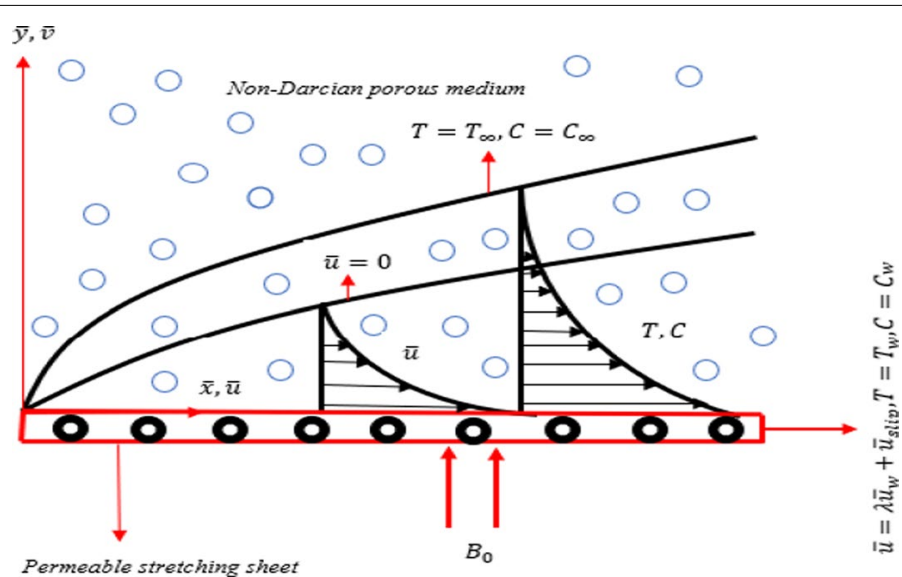

Fig. 1 The physical geometry of the flow 
the fluid temperature and the fraction of nanoparticles in the fluid while the ambient temperature and the nanoparticle volume fraction are described by the symbols $T_{\infty}$ and $C_{\infty}$ respectively. $T_{w}$ stands for the fluid wall temperature and $C_{w}$ stands for the volume of nanoparticles on the wall A magnetic field of strength $B_{0}$ is placed normal to $\bar{x}$. (Fig. 2).

Continuity equation

$$
\frac{\partial \bar{u}}{\partial \bar{x}}+\frac{\partial \bar{v}}{\partial \bar{y}}=0
$$

\section{Momentum equation}

$$
\bar{u} \frac{\partial \bar{u}}{\partial \bar{x}}+\bar{v} \frac{\partial \bar{u}}{\partial \bar{y}}=\frac{\mu_{B}}{\rho_{f}}\left(1+\frac{1}{\beta}\right) \frac{\partial^{2} \bar{u}}{\partial \bar{y}^{2}}-\frac{\sigma_{0} B_{0}^{2}}{\rho_{f}} \bar{u}-\frac{\mu_{B}}{\rho_{f}\left(k_{p}\right)_{o}}\left(1+\frac{1}{\beta}\right) \bar{u}-\frac{b^{*}}{\left(k_{p}\right)_{o}} \bar{u}^{2}
$$

Energy equation

$$
\begin{aligned}
\bar{u} \frac{\partial T}{\partial \bar{x}}+\bar{v} \frac{\partial T}{\partial \bar{y}}= & \alpha \frac{\partial^{2} T}{\partial \bar{y}^{2}}+\tau\left[D_{B} \frac{\partial C}{\partial \bar{y}} \frac{\partial T}{\partial \bar{y}}+\frac{D_{T}}{T_{\infty}}\left(\frac{\partial T}{\partial \bar{y}}\right)^{2}\right] \\
& +\left(1+\frac{1}{\beta}\right) \frac{\mu_{B}}{\left(\rho C_{p}\right)_{f}}\left(\frac{\partial \bar{u}}{\partial \bar{y}}\right)^{2} \\
& +\frac{\sigma B_{0}^{2}}{\left(\rho C_{p}\right)_{f}} \bar{u}^{2}+\left(1+\frac{1}{\beta}\right) \frac{\mu_{B}}{\left(\rho C_{p}\right)_{f}\left(k_{p}\right)_{o}} \bar{u}^{2} \\
& +\frac{b^{*}}{\left(k_{p}\right)_{o}\left(C_{p}\right)_{f}} \bar{u}^{3}
\end{aligned}
$$

\section{Mass conservation}

$$
\bar{u} \frac{\partial C}{\partial \bar{x}}+\bar{v} \frac{\partial C}{\partial \bar{y}}=D_{B} \frac{\partial^{2} C}{\partial \bar{y}^{2}}+\frac{D_{T}}{T_{\infty}} \frac{\partial^{2} T}{\partial \bar{y}^{2}}-K r\left(C-C_{\infty}\right)\left(\frac{T}{T_{\infty}}\right)^{m} e^{\frac{-E_{a}}{\kappa T}}
$$

\section{Entropy generation}

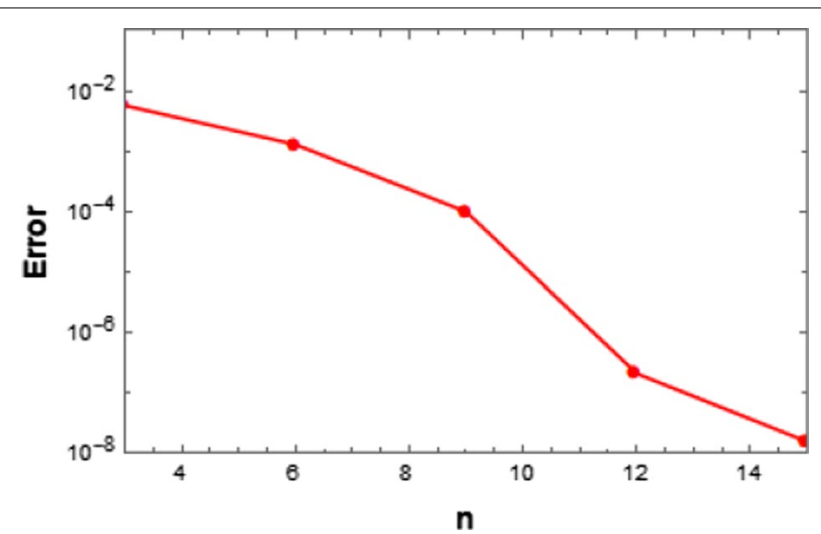

Fig. 2 The maximum average squared residual error at different orders of approximation 


$$
\begin{aligned}
N_{G}= & \frac{K}{T_{\infty}^{2}}\left[\left(\frac{\partial T}{\partial \bar{y}}\right)^{2}\right]+\frac{\mu_{B}}{T_{\infty}}\left(1+\frac{1}{\beta}\right) \\
& +\left[\left(\frac{\partial \bar{u}}{\partial \bar{x}}\right)^{2}+\left(\frac{\partial \bar{v}}{\partial \bar{y}}\right)^{2}\right] \\
& +\frac{\sigma_{0 B_{0}^{2}}}{T_{\infty}}\left(v^{2}+u^{2}\right)+\frac{R D}{C_{\infty}}\left[\left(\frac{\partial \bar{u}}{\partial \bar{x}}\right)^{2}+\left(\frac{\partial \bar{v}}{\partial \bar{y}}\right)^{2}\right] \\
& +\frac{R D}{T_{\infty}}\left[\left(\frac{\partial \bar{u}}{\partial \bar{x}}\right)^{2}+\left(\frac{\partial \bar{v}}{\partial \bar{y}}\right)^{2}\right]+\left[\left(\frac{\partial T}{\partial \bar{y}}\right)\left(\frac{\partial C}{\partial \bar{y}}\right)\right]
\end{aligned}
$$

The corresponding boundary conditions are.

$$
\left\{\begin{array}{l}
\bar{u}=\lambda \bar{u}_{w}(\bar{x})+\bar{u}_{\mathrm{slip}}, \bar{v}=v_{w}, T=T_{w}, C=C_{w}, \text { at } \bar{y}=0 \\
\bar{u} \rightarrow 0, T \rightarrow T_{\infty}, C \rightarrow C_{\infty} \text { as } \bar{y} \rightarrow \infty
\end{array}\right.
$$

The second-order velocity slip that was used is as follows [49].

$$
\bar{u}_{\text {slip }}=\frac{\mu_{B}}{\rho_{f}}\left(1+\frac{1}{\beta}\right)\left[\left(N_{1}\right)_{o} \frac{\partial \bar{u}}{\partial \bar{y}}+\left(N_{2}\right)_{o} \frac{\partial^{2} \bar{u}}{\partial \bar{y}^{2}}\right]
$$

The second-order velocity slip constant factor is $\left(N_{2}\right)_{o}<0$ while first-order velocity slip constant factor is $\left(N_{1}\right)_{o}>0$.

The transformed Arrhenius function is defined by the term $\left(\frac{T}{T_{\infty}}\right)^{m} e^{\frac{-E_{a}}{\kappa T}}$, $\kappa=8.61 \times 10^{-5} \mathrm{eV} / \mathrm{K}$ is Boltzmann constant $m$ is the unitless exponent fitted rate constant $(-1<m<1)$ and $E_{a}$ is the activation energy. The following non-dimensional quantities were introduced:

$$
\begin{aligned}
& \phi=\phi(\eta), b^{*}=\left(b^{*}\right)_{o} x^{-1}, \theta=\theta(\eta), \tau=\frac{\left(\rho C_{p}\right)_{p}}{\left(\rho C_{p}\right)_{f}}, \alpha=\frac{k}{\left(\rho C_{p}\right)_{f}}, v=\frac{\mu_{B}}{\rho_{f}}\left(1+\frac{1}{\beta}\right), \psi=x f(\eta), \\
& \bar{u}_{w}=\frac{U_{r \bar{x}}}{L}, x=\frac{\bar{x}}{L}, y=\frac{\bar{y} \sqrt{\mathrm{Re}}}{L}, u=\frac{\bar{u}}{U_{r}}, v=\frac{\bar{\nu} \sqrt{\operatorname{Re}}}{U_{r}}, \theta=\frac{T-T_{\infty}}{T_{w}-T_{\infty}}, \phi=\frac{C-C_{\infty}}{C_{w}-C_{\infty}}, \operatorname{Pr}=\frac{\nu}{\alpha}, N b=\frac{\tau D_{B}\left(C_{w}-C_{\infty}\right)}{\nu} \\
& S c=\frac{\nu}{D_{B}}, \sigma^{*} \frac{K r L}{U_{r}}, M=\frac{\sigma B_{0}^{2} L}{\rho_{f} U_{r}}, E=\frac{E_{a}}{\kappa T_{\infty}}, P p=\frac{\nu L}{U_{r}\left(k_{p}\right)_{o}}, E c=\frac{u_{w}}{\left(C_{p}\right)_{f}\left(T_{w}-T_{\infty}\right)}, \\
& F s=\frac{\left(b^{*}\right)_{o} L}{\left(k_{p}\right)_{o}}, \delta_{1}=\frac{\left(N_{1}\right)_{o} \mu_{B \sqrt{\mathrm{Re}}}}{\rho_{f} L}(>0), \delta_{2}=\frac{\left(N_{2}\right)_{o} \mu_{B} \operatorname{Re}}{\rho_{f} L^{2}}(<0), S=\frac{v_{w \sqrt{\mathrm{Re}}}}{U_{r}}, u=\frac{\partial \psi}{\partial y}, \nu=-\frac{\partial \psi}{\partial x}
\end{aligned}
$$

are introduced into Eqs. (1)-(8) to obtain the following dimensionless ordinary differential equations.

$$
\begin{aligned}
& \left(1+\frac{1}{\beta}\right) f^{\prime \prime \prime}+f^{\prime \prime} f-f^{\prime 2}-M f^{\prime}-P p\left(1+\frac{1}{\beta}\right) f^{\prime}-F s f^{\prime 2}=0 \\
& \frac{1}{\operatorname{Pr}} \theta^{\prime \prime}+f \theta^{\prime}+N b \theta^{\prime} \phi^{\prime}+N t \theta^{\prime \prime} 2+E c\left(1+\frac{1}{\beta}\right) f^{\prime \prime 2}+E c M f^{\prime 2}+E c P p\left(1+\frac{1}{\beta}\right) f^{\prime 2}+E c F s f^{\prime 3}=0 \\
& \phi^{\prime \prime}+\frac{N t}{N b} \theta^{\prime \prime}+\operatorname{Scf} \phi^{\prime}-\operatorname{Sc} \sigma^{* 2} \phi((T r-1) \theta+1)^{m} e^{\frac{-E}{(T r-1) \theta+1}}=0
\end{aligned}
$$




$$
\begin{aligned}
& N_{1}=\operatorname{Re}(1+N)\left(\theta^{2}+\phi^{2}\right) \\
& N_{2}=\frac{B r}{\Omega}\left(1+\frac{1}{\beta}\right)\left(f^{\prime 2}+\operatorname{Re} f^{\prime \prime 2}\right)+\frac{M \operatorname{Pr}}{\Omega}\left(f+\operatorname{Re} f^{\prime 2}\right)+\lambda\left(\frac{\varepsilon}{\Omega}\right) \theta^{\prime}(\boldsymbol{\eta})+\phi^{\prime}(\boldsymbol{\eta})
\end{aligned}
$$

with the corresponding boundary conditions

$$
\left\{\begin{array}{l}
f(0)=S, f^{\prime}(0)=\lambda+\left(1+\frac{1}{\beta}\right)\left(\delta_{1} f^{\prime \prime}(0)+\delta_{2} f^{\prime \prime \prime}(0)\right), \theta(0)=1, \phi(0)=1 \\
f^{\prime}(\infty) \rightarrow 0, \theta(\infty) \rightarrow 0, \phi(\infty) \rightarrow 0
\end{array}\right.
$$

Other quantities of concern are the bejan number, Nusselt number, and Sherwood number which can be given as

$$
S_{0}=\frac{R(D T)^{2}}{L^{2} T_{\infty}^{2}}, C f_{\bar{x}}=\frac{2 \tau_{w}}{\rho U^{2}}, N u_{\bar{x}}=\frac{-\bar{x}}{T_{w}-T_{\infty}}\left(\frac{\partial T}{\partial \bar{y}}\right)_{\bar{y}=0} \text { and } S h_{\bar{x}}=\frac{-\bar{x}}{C_{w}-C_{\infty}}\left(\frac{\partial C}{\partial \bar{y}}\right)_{\bar{y}=0}
$$

\section{Numerical solution}

\section{A summary of the Chebyshev collocation method}

The spectral system is applied in various fields of engineering to find the numerical solution of several equations (standard, partial, linear, non-linear, and fractional [52]. Chebyshev's collocation approach is more successful in numerical methods like the finite difference methods, with various numerical methods such as finite difference schemes and iterative processes $[53,54]$. In the case of numerical techniques such as final differential schemes, the method of collocating Chebyshev is more effective than numerical techniques like finite and iterative schemes, since in a small number of terminologies the correct solution produces with minimal storage. This approach is better than other numerical methods.

\section{Application of CC}

To apply CCM in this problem, by using suitable transformations, convert the semiinfinite flow of the problem to the Chebyshev polynomial domain $[-1,1]$. Then using the shifted Chebyshev base function from $[-1,1]$ to $[0, \mathrm{~L}]$. The boundary value problem (BVP) in Eqs. (9) and (13) is solved via the Chebyshev collocation scheme (CCS). The description of this method can be found in [24].

$$
f(\eta)=\sum_{i=0}^{N} a_{i} T_{i}\left(\frac{2 \eta}{L}-1\right), \theta(\eta)=\sum_{i=0}^{N} b_{i} T_{i}\left(\frac{2 \eta}{L}-1\right) \text { and } \phi(\eta)=\sum_{i=0}^{N} c_{i} T_{i}\left(\frac{2 \eta}{L}-1\right)
$$

Here, $a_{i}, b_{i}, c_{i}$ are unknown constant coefficients to be determined. $T_{i}\left(\frac{2 \eta}{L}-1\right)$ is the shifted Chebyshev base function from $[-1,1]$ to $[0, L]$ where $L$ stands for the edge of the boundary layer. To compute the values of unknown coefficients, Eqs. (9) and (13) can be transformed as: 


$$
\begin{aligned}
& {\left[\left(1+\frac{1}{\beta}\right) F_{\eta, 4}+F_{\eta, 2} F_{\eta}-F_{\eta}^{2}-M F_{\eta}-P p\left(1+\frac{1}{\beta}\right) F_{\eta}-F s F_{\eta}^{2}\right]_{\eta=\eta_{j}}=0 \text { for } j=1,2, \ldots, N-3,} \\
& \left.\left[\left[\frac{1}{\operatorname{Pr}}\right] \theta_{\eta, \eta}+F \theta_{\eta}^{2}+N b \theta_{\eta} \phi_{\eta}+E c\left(1+\frac{1}{\beta}\right) \theta_{\eta, \eta}^{2}+E c M F_{\eta}^{2}+E c M F_{\eta}^{2}+E c\left(1+\frac{1}{\beta}\right) F_{\eta}^{2} E c F_{\eta, 3}\right]_{\eta=\eta_{j}}\right] \\
& \text { for } j=1,2, \ldots, N-1, \\
& {\left[\left[\phi_{\eta, \eta}+\frac{N t}{N b} \theta_{\eta, \eta}+s c F \phi_{\eta}-S c \sigma^{*^{2}} \phi((T r-1) \theta+1)^{m} e^{\frac{-E}{(T r-1) \theta+1}}\right]\right]_{\eta=\eta_{j}}} \\
& \text { for } j=1,2, \ldots, N-1,]_{\eta=L} \\
& {\left[\frac{\mathrm{d}}{\mathrm{d} \eta} \sum_{i=0}^{N} a_{i} T_{i}\left(\frac{2 \eta}{L}-1\right)\right]_{\eta=L}=0,\left[\sum_{i=0}^{N} b_{i} T_{i}\left(\frac{2 \eta}{L}-1\right)-1\right]_{\eta=0}} \\
& =0,\left[\sum_{i=0}^{N} c_{i} T_{i}\left(\frac{2 \eta}{L}-1\right)-1\right]_{\eta=0}=0
\end{aligned}
$$

$R_{f}\left(\eta, a_{i}, b_{i}\right), R_{\theta}\left(\eta, a_{i}, b_{i}, c_{i}\right)$ and $R_{\phi}\left(\eta, a_{i}, b_{i}, c_{i}\right)$ are derived by substituting Eq. (15) into (16)-(18). The residues are minimized as small as possible using the collocation method as follows:

$$
\begin{aligned}
& \text { For } \delta\left(\eta-\eta_{k}\right)=\left\{\begin{array}{l}
1, \eta=\eta_{j} \\
0, \text { otherwise, }
\end{array}\right. \\
& \int_{0}^{L} R_{f} \delta\left(\eta-\eta_{j}\right) \mathrm{d} \eta=R_{f}\left(\eta_{j}\right)=0, \quad \text { for } j=1,2, \ldots N-2 \\
& \int_{0}^{L} R_{\theta} \delta\left(\eta-\eta_{j}\right) \mathrm{d} \eta=R_{\theta}\left(\eta_{j}\right)=0, \quad \text { for } j=1,2, \ldots N-1 \\
& \int_{0}^{L} R_{\phi} \delta\left(\eta-\eta_{j}\right) \mathrm{d} \eta=R_{\phi}\left(\eta_{j}\right)=0, \quad \text { for } j=1,2, \ldots N-1
\end{aligned}
$$

where $\eta_{j}$ is the shifted Gauss Lobato collocation points defined as follows

$$
\eta_{j}=\frac{1}{2}\left(1-\cos \left(\frac{j \pi}{N}\right)\right), \text { for } j=0,1, \ldots N
$$

In this manner, Eqs. (16)-(18) form a system of $3 N+3$ algebraic equations with $3 N+3$ unknown constant coefficients, $\left(a_{i} b_{i}\right.$ and $\left.c_{j}\right)$. The derived algebraic equations are solved using the Newton method and the values of constant coefficients are obtained. The value of $\mathrm{L}$ is chosen to be 12 to ensure that all numerical solutions obey the far-field asymptotic correctly.

\section{Solution by optimal homotopy analysis method (OHAM)}

The governing boundary value problem (BVP) is described by Eqs. (9) and (13). These equations are highly nonlinear ordinary differential equations that define the governing boundary value problem (BVP). According to the existing literature, such BVP can therefore be resolved with different techniques. which include perturbation method, The weighted residual method [36, 37], finite difference method [38], finite different element 
[39], Runge-Kutta scheme [40]. The solution of these non-linear (BVP) continues to fascinate and motivate scientists to create methods for obtaining solutions that explain the complex characteristics of the underlying problem of boundary layer flow under various conditions $[55,56]$. However, the optimal homotopy analysis approach (OHAM) is used in this study. It is based on the homotopy principle, which is a fundamental idea in differential geometry and topology. The special qualities which distinguish OHAM from all other methods (especially analytic approximate methods) are.

1. It provides freedom in the selection of the auxiliary linear operator, which distinguishes OHAM from all other methods (especially analytic approximate methods). The initial guess, form of the equation, and solution expression of high-order equations; therefore, approximations at very high-order are easy to obtain.

2. It provides a very simple way to ensure solution series convergence.

3. Furthermore, unlike HAM, the square residual error minimization is used to find an optimum value for the auxiliary parameter $h$. Because of this unique quality, OHAM has effectively solved several non-linear engineering, scientific, financial, etc. issues.

This method is defined in detail in [57]. OHAM is used to solve equations (9) and (13), which are subject to boundary conditions The original guesses and the linear auxiliary operator as follow:

$$
L_{f}=\frac{\mathrm{d}^{2}}{\mathrm{~d} \eta^{2}}, L_{\theta}=\frac{\mathrm{d}^{2}}{\mathrm{~d} \eta^{2}}, L_{\phi}=\frac{\mathrm{d}^{2}}{\mathrm{~d} \eta^{2}}
$$

Together with the properties

$$
L_{f}\left(c_{1}+c_{2} y\right)=0, L_{\theta}\left(c_{3}+c_{4} y\right)=0, L_{\phi}\left(c_{5}+c_{6} y\right)=0
$$

To decide the arbitrary constants of the boundary conditions, we utilized $c_{j}(j=1 \cdots 6)$, for $p \in[0,1]$. The deformation problem of zeroth-order can be described as

$$
\begin{gathered}
(1-p) L_{f}\left[\hat{u}(\eta ; p)-u_{f}(\eta)\right]=p \hbar_{f} \aleph_{f}[\hat{f}(\eta ; p), \hat{\theta}(\eta ; p), \hat{\phi}(\eta ; p)] \\
(1-p) L_{\theta}\left[\hat{\theta}(\eta ; p)-\theta_{0}(\eta)\right]=p \hbar_{\theta} \aleph_{\theta}[\hat{f}(\eta ; p), \hat{\theta}(\eta ; p), \hat{\phi}(\eta ; p)] \\
(1-p) L_{\phi}\left[\hat{\phi}(\eta ; p)-\phi_{0}(\eta)\right]=p \hbar_{\phi} \aleph_{\phi}[\hat{f}(\eta ; p), \hat{\theta}(\eta ; p), \hat{\phi}(\eta ; p)] \\
\aleph_{f}[\hat{f}(\eta ; p), \hat{\theta}(\eta ; p), \hat{\phi}(\eta ; p)]=\left(1+\frac{1}{\beta}\right) \frac{\partial^{3} \hat{f}(\eta ; p)}{\partial \eta^{3}}+\hat{f}(\eta ; p) \frac{\partial^{2} \hat{f}(\eta ; p)}{\partial \eta^{2}} \\
-\left(\frac{\partial \hat{f}(\eta ; p)}{\partial \eta^{2}}\right)^{2}-P p\left(1+\frac{1}{\beta}\right)\left(\frac{\partial \hat{f}(\eta ; p)}{\partial \eta}\right)-F s\left(\frac{\partial \hat{f}(\eta ; p)}{\partial \eta^{2}}\right)^{2}
\end{gathered}
$$




$$
\begin{aligned}
& \aleph_{\theta}[\hat{u}(y ; p), \hat{\theta}(y ; p), \hat{\phi}(y ; p)] \\
& =\frac{1}{\operatorname{Pr}}\left(\frac{\partial^{2} \hat{\theta}(\eta ; p)}{\partial \eta^{2}}\right)+\hat{f}(\eta ; p)\left(\frac{\partial \hat{\theta}(\eta ; p)}{\partial \eta}\right) \\
& \quad+N b\left(\frac{\partial \hat{\theta}(\eta ; p)}{\partial \eta}\right)\left(\frac{\partial \hat{\phi}(\eta ; p))}{\partial \eta}\right)+N t\left(\frac{\partial \hat{\theta}(\eta ; p)}{\partial \eta^{2}}\right)^{2} \\
& \quad+E c\left(1+\frac{1}{\beta}\right)\left(\frac{\partial^{2} \hat{f}(\eta ; p)}{\partial \eta^{2}}\right)^{2} \\
& =E c M\left(\frac{\partial \hat{f}(\eta ; p)}{\partial \eta^{2}}\right)^{2}+E c P p\left(1+\frac{1}{\beta}\right)\left(\frac{\partial \hat{f}(\eta ; p)}{\partial \eta^{2}}\right)^{2} \\
& +E c F s\left(\frac{\partial^{3} \hat{f}(\eta ; p)}{\partial \eta^{3}}\right) \quad-S c \sigma^{*^{2}} \hat{\phi}(\eta ; p)((T r-1) \hat{\theta}(\eta ; p)+1)^{m} e^{\frac{-E}{(T r-1) \hat{\theta}(\eta ; p)+1}} \\
& \aleph_{\phi}[\hat{f}(\eta ; p), \hat{\theta}(\eta ; p), \hat{\phi}(\eta ; p)]=\frac{\partial^{2} \hat{\phi}(\eta ; p)}{\partial \eta^{2}}+\frac{N t}{N b}\left(\frac{\partial^{2} \hat{\theta}(\eta ; p)}{\partial \eta^{2}}\right) \\
& \quad+S c\left(\hat{f}(\eta ; p) \frac{\partial \hat{\phi}(\eta ; p)}{\partial \eta}\right)
\end{aligned}
$$

as a function of the boundary conditions

$$
\begin{aligned}
& \hat{f}(0 ; p)=S, \frac{\partial \hat{f}(0 ; p)}{\partial \eta}=\lambda+\left(1+\frac{1}{\beta}\right)\left(\delta_{1}\left(\frac{\partial^{2} \hat{f}(0 ; p)}{\partial \eta^{2}}\right)+\delta_{2} \frac{\partial^{3} \hat{f}(0 ; p)}{\partial \eta^{3}}\right), \\
& \hat{\theta}(0 ; p)=1, \hat{\phi}(0 ; p=1 \\
& \frac{\partial \hat{f}(\infty ; p)}{\partial \eta} \rightarrow 0, \hat{\theta}(\infty ; p) \rightarrow 0, \hat{\phi}(\infty ; p) \rightarrow 0,
\end{aligned}
$$

where $\aleph_{f}, \aleph_{\theta}$ and $\aleph_{\phi}$ are nonlinear operators and $\hbar_{f}, \hbar_{\theta}$ and $\hbar_{\phi}$ are nonzero auxiliary parameters. It gives the following results for $p=0$ and $p=1$

$$
\begin{aligned}
& \hat{f}(\eta ; 0)=u_{0}(\eta), \hat{f}(\eta ; 1)=u(\eta), \\
& \hat{\theta}(\eta ; 0)=\theta_{0}(\eta), \hat{\theta}(\eta ; 1)=\theta(\eta), \\
& \hat{\phi}(\eta ; 0)=\phi_{0}(\eta y), \hat{\phi}(\eta ; 1)=\phi(\eta)
\end{aligned}
$$

In other words, when $\mathrm{p}$ varies from 0 to 1 , then $\hat{f}(\eta ; p), \hat{\theta}(\eta ; p)$ and $\hat{\phi}(\eta ; p)$ vary from $f_{0}(\eta), \theta_{0}(\eta)$ and $\phi_{0}(\eta)$ to $f(\eta), \theta(\eta)$ and $\phi(\eta)$. Now, the Taylor series expansion of $\hat{f}(\eta ; p), \hat{\theta}(\eta ; p)$ and $\hat{\phi}(\eta ; p)$ concerning $p$ yield where 


$$
\begin{aligned}
& f(n, p)=u_{0}(\eta)+\sum_{m=1}^{\infty} u_{m}(\eta) p^{m} \\
& \theta(n, p)=\theta_{0}(\eta)+\sum_{m=1}^{\infty} \theta_{m}(\eta) p^{m} \\
& \phi(n, p)=\phi_{0}(\eta)+\sum_{m=1}^{\infty} \phi_{m}(\eta) p^{m} \\
& f_{m}(\eta)=\left.\frac{1}{m !} \frac{\partial^{m} f(\eta ; p)}{\partial y^{m}}\right|_{p=0}, \\
& \theta_{m}(\eta)=\left.\frac{1}{m !} \frac{\partial^{m} \theta(\eta ; p)}{\partial y^{m}}\right|_{p=0}, \\
& \phi_{m}(\eta)=\left.\frac{1}{m !} \frac{\partial^{m} \phi(\eta ; p)}{\partial y^{m}}\right|_{p=0}
\end{aligned}
$$

It can be obtained if the auxiliary parameters, initial guesses, and auxiliary linear operators are correctly chosen such that Eqs. (24)-(26) converge at $p=1$.

$$
\begin{aligned}
& f(\eta)=u_{0}(\eta)+\sum_{m=1}^{\infty}, u_{m}(\eta), \\
& \theta(\eta)=\theta_{0}(\eta)+\sum_{m=1}^{\infty}, \theta_{m}(y), \\
& \phi(\eta)=\phi_{0}(\eta)+\sum_{m=1}^{\infty}, \phi_{m}(y),
\end{aligned}
$$

The corresponding $m$ th order deformation problems are:

$$
\begin{aligned}
& L_{f}\left[f_{m}(\eta)-X_{m} u_{m-1}\right]=\hbar_{u} R_{u, m}(\eta), \\
& L_{\theta}\left[\theta_{m}(\eta)-X_{m} \theta_{m-1}\right]=\hbar_{\theta} R_{\theta, m}(\eta), \\
& L_{\phi}\left[\phi_{m}(\eta)-X_{m} \phi_{m-1}\right]=\hbar_{\phi} R_{\phi, m}(\eta), \\
& f_{m}(0)=S, f_{m}^{\prime}(0)=\lambda+\left(1+\frac{1}{\beta}\right)\left(\delta_{1} f_{m}^{\prime \prime}(0)+\delta_{2} f_{m}^{\prime \prime \prime}(0)\right), \phi_{m}(0)=1, \phi_{m}(0)=1 \\
& f_{m}^{\prime}(\infty) \rightarrow 0, \theta_{m}(\infty) \rightarrow 0, \phi_{m}(\infty) \rightarrow 0,
\end{aligned}
$$

subjected to boundary conditions

$$
\begin{aligned}
R_{f, m}(y)= & \left(1+\frac{1}{\beta}\right) \frac{\partial^{3} \hat{f}_{m-j-1}(\eta)}{\partial \eta^{3}}+\sum_{k=0}^{m-1}\left(\hat{f}(\eta) \frac{\partial^{3} \hat{f}_{m-j-1}(\eta)}{\partial \eta^{3}}-\frac{\partial \hat{f}_{j}(\eta)}{\partial \eta} \frac{\partial \hat{f}_{m-j-1}(\eta)}{\partial \eta}+m \frac{\partial^{2} \hat{f}_{m-j-1}(\eta)}{\partial \eta^{2}}\right. \\
& \left.+P p\left(1+\frac{1}{\beta}\right) \frac{\partial^{2} \hat{f}_{m-j-1}(\eta)}{\partial \eta^{2}}-F s \frac{\partial^{2} \hat{f}_{j}(\eta)}{\partial \eta^{2}} \frac{\partial \hat{f}_{m-j-1}(\eta)}{\partial \eta}\right)
\end{aligned}
$$


Table 1 SCM solutions in various approximation orders of convergence when $M=P p=1=2=\beta=1, F s=\delta=\delta_{1}=\delta_{2}=0.5$

\begin{tabular}{llll}
\hline Number of iteration $(\boldsymbol{N})$ & $-\boldsymbol{f}^{\prime \prime}(\boldsymbol{\eta})$ & $-\boldsymbol{\theta}^{\prime}(\mathbf{0})$ & $\boldsymbol{\varphi}^{\prime}(\mathbf{0})$ \\
\hline 4 & 3.054620 & 0.233124 & 0.358571 \\
6 & 3.004633 & 0.180744 & 0.297306 \\
8 & 3.002122 & 0.98752 & 0.06982 \\
10 & 3.001933 & 0.987525 & 0.06982 \\
12 & 3.001933 & 0.98752 & 0.06982 \\
14 & 3.001933 & 0.180557 & 0.296766 \\
16 & 3.001933 & 0.180557 & 0.296766 \\
20 & 3.001933 & 0.180557 & 0.296766 \\
24 & 3.001933 & 0.180557 & 0.296766 \\
30 & 3.001933 & 0.180557 & 0.296766 \\
\hline
\end{tabular}

Table 2 Show the optimal auxiliary parameter values at various approximation values when $M=P p=1=2=\beta=1, F s=\delta=\delta_{1}=\delta_{2}=0.5$

\begin{tabular}{lllll}
\hline Order-of approximation & $\hbar_{\boldsymbol{f}}$ & $\hbar_{\boldsymbol{\theta}}$ & $\hbar_{\boldsymbol{\phi}}$ & $\boldsymbol{\Delta}_{\boldsymbol{m}}^{\boldsymbol{t}}$ \\
\hline 2 & -0.86345 & -2.52072 & -1.51245 & $7.7 \times 10^{-3}$ \\
3 & -0.88412 & -1.92653 & -1.90756 & $2.3 \times 10^{-3}$ \\
4 & -0.89635 & -1.99556 & -1.61023 & $8.5 \times 10^{-3}$ \\
5 & -0.92745 & -1.85853 & -1.96075 & $5.3 \times 10^{-3}$ \\
\hline
\end{tabular}

$$
\begin{aligned}
& R_{\theta, m}(\eta)= \frac{1}{\operatorname{Pr}} \frac{\partial^{2} \hat{\theta}_{m-1}(\eta)}{\partial \eta^{2}}+\sum_{k=0}^{m-1} \hat{f}_{j}(\eta) \frac{\partial \theta_{m-j-1}(\eta)}{\partial \eta} \\
&+N b \frac{\partial \hat{\theta}_{j}(\eta)}{\partial \eta} \frac{\partial \hat{\theta}_{m-j-1}(\eta)}{\partial \eta}+N t \frac{\partial \hat{\theta}_{j}(\eta)}{\partial \eta} \frac{\partial \hat{\theta}_{m-j-1}(\eta)}{\partial \eta} \\
&+E c\left(1+\frac{1}{\beta}\right) \frac{\partial \hat{f}_{j}(\eta)}{\partial \eta} \frac{\partial \hat{f}_{m-j-1}(\eta)}{\partial \eta}+E c M \frac{\partial \hat{f}_{j}(\eta)}{\partial \eta} \frac{\partial \hat{f}_{m-j-1}(\eta)}{\partial \eta} \\
&+E c P p\left(1+\frac{1}{\beta}\right) \frac{\partial^{2} \hat{f}_{j}(\eta)}{\partial \eta^{2}} \frac{\partial \hat{f}_{m-j-1}(\eta)}{\partial \eta}+E c F s \\
& \frac{\partial^{2} \hat{f}_{j}(\eta)}{\partial \eta^{2}} \frac{\partial \hat{f}_{m-j-1}(\eta)}{\partial \eta}, \\
& R_{\phi, m}(\eta)= \frac{\partial^{2} \phi_{m-1}(\eta)}{\partial \eta^{2}} \\
&+\sum_{k=0}^{m-1}\left(\frac{N t}{N b} \frac{\partial \hat{\theta}_{j}(\eta)}{\partial \eta} \frac{\partial \phi_{m-j-1}(\eta)}{\partial \eta}+S c \widehat{f}_{j}(\eta) \frac{\partial \phi_{m-j-1}(\eta)}{\partial \eta}+S c \sigma^{* 2} \phi(\eta)((T r-1) \theta(\eta)+1)^{m} e^{\frac{-E}{(T r-1) \hat{\theta}(\eta)+1}}\right.
\end{aligned}
$$

where

$$
X_{m} \begin{cases}1 & m>1 \\ 0 & m \leq 1\end{cases}
$$

The following are the general solutions: 
Table 3 Show Individual residual square error and OHAM solutions convergence at various approximation orders when $M=P P=1=2=\beta=1, F_{S}=\delta=\delta_{1}=\delta_{2}=0.5, \hbar_{u}=-0.82706, \hbar_{\theta}=-0.75851, \hbar_{\phi}=-0.96084$

\begin{tabular}{llllllll}
\hline $\boldsymbol{m}$ & $\boldsymbol{\Delta}_{\boldsymbol{m}}^{\boldsymbol{f}}$ & $\boldsymbol{\Delta}_{\boldsymbol{m}}^{\boldsymbol{\theta}}$ & $\boldsymbol{\Delta}_{\boldsymbol{m}}^{\boldsymbol{\phi}}$ & $\boldsymbol{f}^{\prime \prime}(\mathbf{0})$ & $\boldsymbol{- \theta}^{\prime}(\mathbf{0})$ & $-\boldsymbol{\phi}^{\prime}(\mathbf{0})$ & CPU time (s) \\
\hline 2 & $1.79 \times 10^{-3}$ & $2.64 \times 10^{-6}$ & $2.68 \times 10^{-8}$ & 2.289542 & 0.137041 & 0.011381 & 0.3437448 \\
4 & $2.54 \times 10^{-8}$ & $1.65 \times 10^{-7}$ & $1.14 \times 10^{-13}$ & 2.273865 & 0.13685 & 0.011487 & 0.7187314 \\
6 & $2.35 \times 10^{-11}$ & $4.85 \times 10^{-11}$ & $9.47 \times 10^{-15}$ & 2.274001 & 0.137146 & 0.011488 & 1.1405919 \\
8 & $1.54 \times 10^{-12}$ & $1.03 \times 10^{-13}$ & $2.26 \times 10^{-17}$ & 2.274003 & 0.137151 & 0.011488 & 1.671824 \\
10 & $8.95 \times 10^{-17}$ & $4.03 \times 10^{-17}$ & $6.25 \times 10^{-20}$ & 2.274004 & 0.137151 & 0.011488 & 2.3905487 \\
12 & $9.20 \times 10^{-19}$ & $3.19 \times 10^{-19}$ & $5.55 \times 10^{-23}$ & 2.274004 & 0.137151 & 0.011488 & 3.3280166 \\
14 & $2.09 \times 10^{-22}$ & $4.05 \times 10^{-22}$ & $1.32 \times 10^{-25}$ & 2.274004 & 0.137151 & 0.011488 & 3.7185883 \\
20 & $7.76 \times 10^{-24}$ & $1.41 \times 10^{-24}$ & $2.63 \times 10^{-28}$ & 2.274004 & 0.137151 & 0.011488 & 12.1245886 \\
26 & $6.19 \times 10^{-27}$ & $1.60 \times 10^{-27}$ & $8.66 \times 10^{-31}$ & 2.274004 & 0.137151 & 0.011488 & 18.2174761 \\
30 & $2.46 \times 10^{-29}$ & $5.56 \times 10^{-30}$ & $1.37 \times 10^{-33}$ & 2.274004 & 0.137151 & 0.011488 & 22.3578236 \\
\hline
\end{tabular}

Table 4 Comparison of wall heat transfer rate $\left(-\theta^{\prime}(0)\right)$ in the absence of nanoparticle for different values of $\operatorname{Pr}$ when $M=P p=F S=S=\delta_{1}=\delta_{2}=0, \beta \rightarrow \infty$

\begin{tabular}{lllll}
\hline Pr & Present Study & Wang [57] & Khan and Pop [60] & $\begin{array}{l}\text { Makinde } \\
\text { and Aziz } \\
\text { [61] }\end{array}$ \\
\hline 0.2 & 0.1734 & 0.1691 & 0.1691 & 0.1691 \\
0.7 & 0.4539 & 0.4539 & 0.4539 & 0.4539 \\
2.0 & 0.9114 & 0.9113 & 0.9114 & 0.9114 \\
\hline
\end{tabular}

$$
\begin{aligned}
& L_{f}(\eta)=f_{m}^{*}(\eta)+c_{1}+c_{2} \eta, \\
& \theta_{m}(\eta)=\theta_{m}^{*}(\eta)+c_{3}+c_{4} \eta, \\
& \phi_{m}(\eta)=\phi_{m}^{*}(\eta)+c_{5}+c_{6} \eta,
\end{aligned}
$$

then $f_{m}^{*}(\eta), \theta_{m}^{*}(\eta), \phi_{m}^{*}(\eta)$ are the particular solutions $[58,59]$

\section{Convergence control parameter}

It's worth noting that the sequence solution arrangements (38)-(40) provide auxiliary parameters, $\hbar_{f}, \hbar_{\theta}$ and $\hbar_{\phi}$. The degree of convergence solution is prescribed by the various values of the auxiliary parameters of the over-the-level portion of the bends segment of the curves in OHAM. A square residual error reduction is assumed to achieve ideal optimal values for auxiliary parameters as follows:

$$
\begin{aligned}
& \Delta_{m}^{f}=\int_{0}^{l} \aleph_{u}\left(\sum_{k=0}^{m} f\left(\eta, \hbar_{f}, \hbar_{\theta}, \hbar_{\phi}\right), \sum_{k=0}^{m} \theta\left(\eta, \hbar_{u}, \hbar_{\theta}, \hbar_{\phi}\right), \sum_{k=0}^{m} \phi\left(\eta, \hbar_{\theta}, \hbar_{\phi}\right)\right) d \eta, \\
& \Delta_{m}^{f}=\int_{0}^{l} \aleph_{f} \sum_{k=0}^{m} f\left(y, \hbar_{u}, \hbar_{\theta}, \hbar_{\phi}\right), \sum_{k=0}^{m} \theta\left(y, \hbar_{u}, \hbar_{\theta}, \hbar_{\phi}\right), \sum_{k=0}^{m} \phi\left(\eta, \hbar_{\theta}, \hbar_{\phi}\right) \mathrm{d} \eta,
\end{aligned}
$$


Table 5 Values of Nusselt number and Sherwood number for various parameters

\begin{tabular}{|c|c|c|c|c|c|}
\hline \multirow[t]{2}{*}{ Parameters } & \multirow[t]{2}{*}{ Values } & \multicolumn{2}{|l|}{$\theta^{\prime}(\eta)$} & \multicolumn{2}{|l|}{$-\phi^{\prime}(\eta)$} \\
\hline & & OHAM & CCS & OHAM & CCS \\
\hline \multirow[t]{3}{*}{ M } & 0.1 & 3.230213 & 3.230214 & 3.3704865 & 3.3704866 \\
\hline & 0.3 & 3.259212 & 3.259213 & 3.3883765 & 3.3883766 \\
\hline & 0.5 & 3.2682832 & 3.2682833 & 3.4036234 & 3.4036235 \\
\hline \multirow[t]{3}{*}{$P p$} & 0.1 & 3.230721 & 3.230722 & 3.370476 & 3.370474 \\
\hline & 0.3 & 3.223712 & 3.223713 & 3.373123 & 3.373122 \\
\hline & 0.5 & 3.219021 & 3.219022 & 3.377754 & 3.377753 \\
\hline \multirow[t]{3}{*}{ EC } & 0.1 & 3.230932 & 3.230933 & 3.370645 & 3.370644 \\
\hline & 0.3 & 3.494832 & 3.494831 & 3.564124 & 3.564125 \\
\hline & 0.5 & 3.773123 & 3.773122 & 3.770543 & 3.770542 \\
\hline \multirow[t]{3}{*}{$\beta$} & 0.1 & 3.230832 & 3.230831 & 3.370124 & 3.370125 \\
\hline & 0.3 & 3.251234 & 3.251235 & 3.208234 & 3.2082355 \\
\hline & 0.5 & 3.272883 & 3.272882 & 3.087543 & 3.087544 \\
\hline \multirow[t]{3}{*}{$\mathrm{Nb}$} & 0.1 & 3.230234 & 3.230231 & 3.370123 & 3.370122 \\
\hline & 0.3 & 3.013844 & 3.013843 & 3.073123 & 3.073123 \\
\hline & 0.5 & 2.868346 & 2.868345 & 2.878232 & 3.878233 \\
\hline \multirow[t]{3}{*}{$N t$} & 0.1 & 1.224089 & 1.224089 & 1.364179 & 1.364178 \\
\hline & 0.5 & 1.225770 & 1.225770 & 1.365968 & 1.3659669 \\
\hline & 1.0 & 1.227898 & 1.227898 & 1.368220 & 1.3682121 \\
\hline
\end{tabular}

Table 6 Skin friction coefficient and rate of heat transfer for (Prandtl-number $=7$ Water)

\begin{tabular}{lllllll}
\hline $\boldsymbol{\beta}$ & Ec & M & $\boldsymbol{P p}$ & $\boldsymbol{F s}$ & $\begin{array}{l}\text { Skin friction } \\
\text { coefficient }\end{array}$ & Heat transfer rate \\
\hline 0.1 & 0.6 & 1.5 & 2 & 0.2 & 2.78522 & 4.78326 \\
1.5 & 0.6 & 1.5 & 2 & 0.2 & 3.42345 & 6.92976 \\
2.0 & 0.6 & 1.5 & 2 & 0.2 & 4.45087 & 7.73098 \\
0.1 & 0.6 & 1.5 & 2 & 0.2 & 3.21246 & 4.83235 \\
0.1 & 2.0 & 1.5 & 2 & 0.2 & 2.88234 & 3.74832 \\
0.1 & 4.0 & 1.5 & 2 & 0.2 & 2.72986 & 3.84978 \\
0.1 & 0.6 & 1.5 & 2 & 0.2 & 3.16234 & 4.97235 \\
0.1 & 0.6 & 2.5 & 2 & 0.2 & 2.65081 & 3.77085 \\
0.1 & 0.6 & 5.0 & 2 & 0.2 & 2.13279 & 4.95124 \\
0.1 & 0.6 & 1.5 & 2 & 0.2 & 3.13064 & 4.49096 \\
0.1 & 0.6 & 1.5 & 7 & 0.2 & 2.25274 & 4.08245 \\
0.1 & 0.6 & 1.5 & 8 & 0.2 & 2.66289 & 2.27092 \\
0.1 & 0.6 & 1.5 & 2 & 0.2 & 2.760411 & 3.67245 \\
0.1 & 0.6 & 1.5 & 2 & 1.0 & 2.642715 & 3.54663 \\
0.1 & 0.6 & 1.5 & 2 & 3.5 & 2.362795 & 2.32023 \\
\hline
\end{tabular}

$$
\Delta_{m}^{\theta}=\int_{0}^{l} \aleph_{\theta} \sum_{k=0}^{m} f\left(\eta, \hbar_{u}, \hbar_{\theta}, \hbar_{\phi}\right), \sum_{k=0}^{m} \theta\left(\eta, \hbar_{u}, \hbar_{\theta}, \hbar_{\phi}\right), \sum_{k=0}^{m} \phi\left(\eta, \hbar_{\theta}, \hbar_{\phi}\right) \mathrm{d} \eta
$$


Table 7 Wall shear stress and rate of heat transfer for (Prandtl-number $=0.71$ Air)

\begin{tabular}{lllllll}
\hline $\boldsymbol{\beta}$ & $\boldsymbol{E}_{\mathrm{c}}$ & $\boldsymbol{M}$ & $\boldsymbol{P p}$ & $\boldsymbol{F s}$ & $\begin{array}{l}\text { Shear stress at the } \\
\text { wall }\end{array}$ & Heat transfer rate \\
\hline 0.1 & 0.6 & 1.5 & 2 & 0.2 & 2.27598 & 6.16312 \\
1.5 & 0.6 & 1.5 & 2 & 0.2 & 3.78987 & 8.24287 \\
2.0 & 0.6 & 1.5 & 2 & 0.2 & 5.82123 & 9.34184 \\
0.1 & 0.6 & 1.5 & 2 & 0.2 & 2.94634 & 3.28432 \\
0.1 & 2.0 & 1.5 & 2 & 0.2 & 2.68943 & 4.17023 \\
0.1 & 4.0 & 1.5 & 2 & 0.2 & 2.47223 & 5.28125 \\
0.1 & 0.6 & 1.5 & 2 & 0.2 & 2.72176 & 5.8486 \\
0.1 & 0.6 & 2.5 & 2 & 0.2 & 2.42124 & 4.44298 \\
0.1 & 0.6 & 5.0 & 2 & 0.2 & 2.08505 & 3.13409 \\
0.1 & 0.6 & 1.5 & 2 & 0.2 & 2.16323 & 6.43025 \\
0.1 & 0.6 & 1.5 & 7 & 0.2 & 2.38184 & 5.48509 \\
0.1 & 0.6 & 1.5 & 8 & 0.2 & 2.3982 & 3.51012 \\
0.1 & 0.6 & 1.5 & 2 & 0.2 & 2.88698 & 7.70609 \\
0.1 & 0.6 & 1.5 & 2 & 1.0 & 2.78154 & 8.28195 \\
0.1 & 0.6 & 1.5 & 2 & 3.5 & 2.45432 & 5.13412 \\
\hline
\end{tabular}

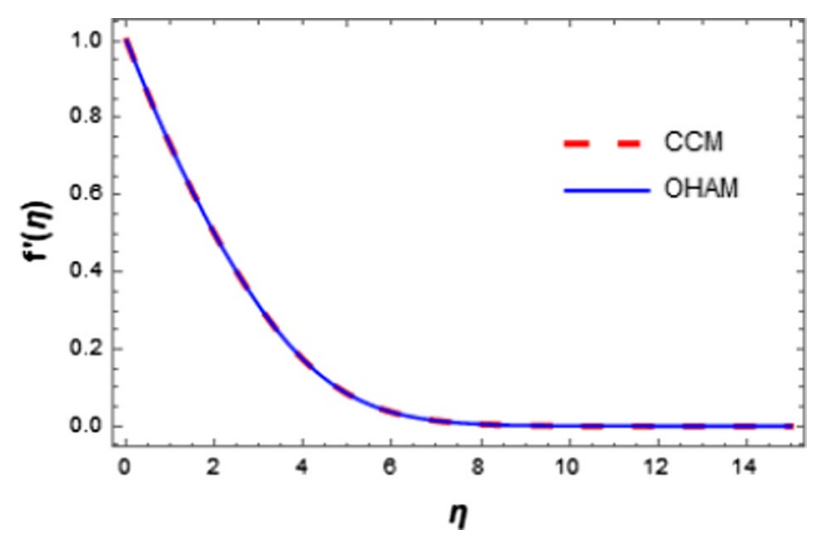

Fig. 3 Comparison of the velocity profile obtained using and CCM and OHAM

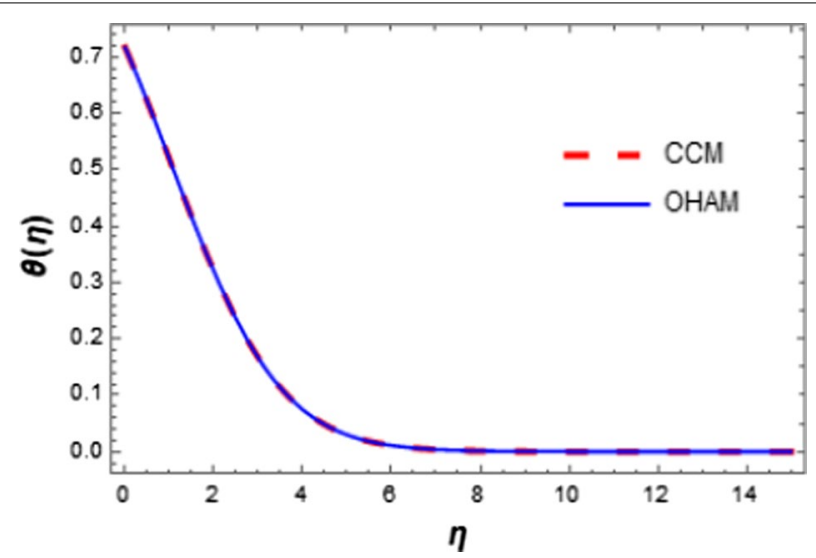

Fig. 4 Comparison of the temperature profile obtained using and CCM and OHAM 


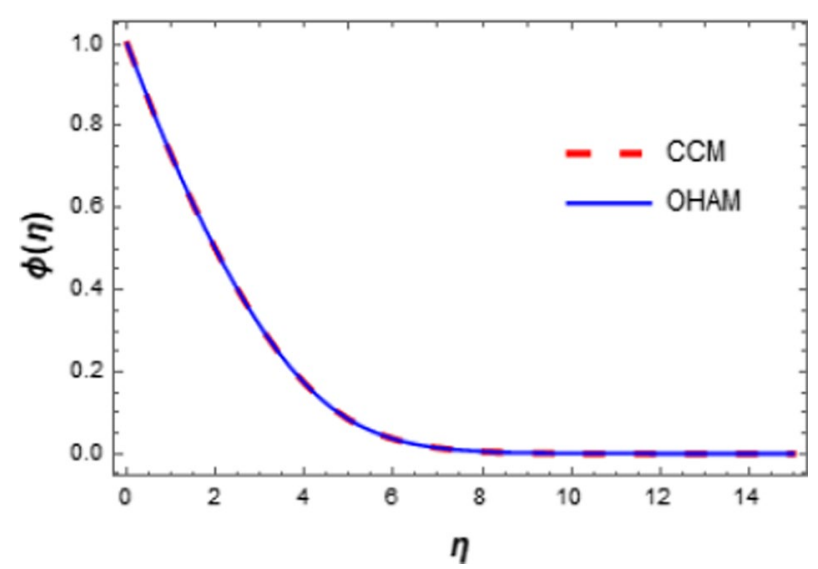

Fig. 5 Comparison of the nanoparticle volume fraction obtained using CCM and OHAM
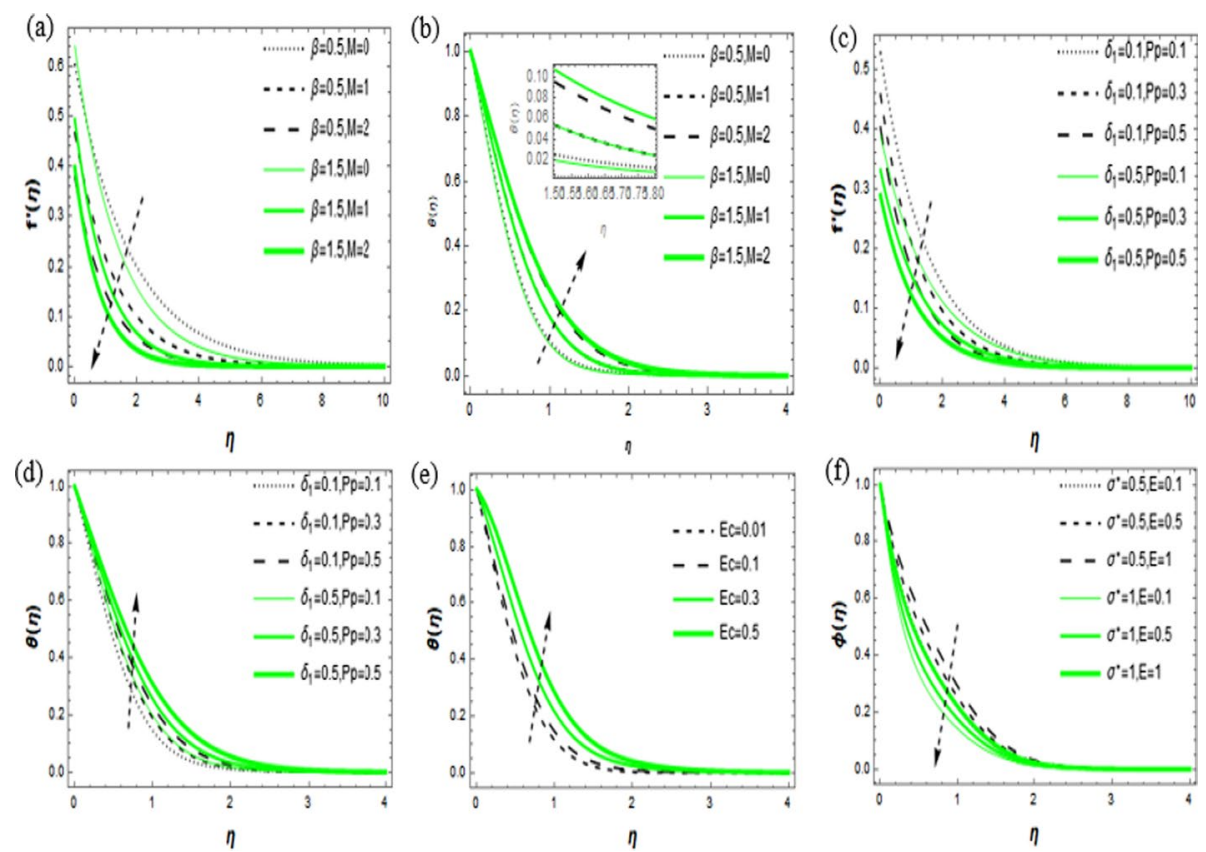

Fig. 6 a, b, c, d, e, and $\mathbf{f}$ Effect of Casson parameter, magnetic field parameter, porosity and first-order slip parameters, second-order slip, wall transpiration (suction/injection) Eckert number, chemical reaction and activation energy parameters on velocity, fluid temperature, amd concentration profiles

$$
\begin{aligned}
\Delta_{m}^{f} & =\int_{0}^{l} \aleph_{f} \sum_{k=0}^{l} f\left(y, \hbar_{u}, \hbar_{\theta}, \hbar_{\phi}\right), \sum_{k=0}^{m} \theta\left(y, \hbar_{u}, \hbar_{\theta}, \hbar_{\phi}\right), \sum_{k=0}^{m} \phi\left(\eta, \hbar_{\theta}, \hbar_{\phi}\right) \mathrm{d} \eta \\
\Delta_{m}^{\theta} & =\int_{0}^{l} \aleph_{\theta} \sum_{0}^{m} f\left(y, \hbar_{u}, \hbar_{\theta}, \hbar_{\phi}\right), \sum_{k=0}^{m} \theta\left(y, \hbar_{u}, \hbar_{\theta}, \hbar_{\phi}\right), \sum_{k=0}^{m} \phi\left(\eta, \hbar_{\theta}, \hbar_{\phi}\right) \mathrm{d} \eta,
\end{aligned}
$$




$$
\Delta_{m}^{\theta}=\int_{0}^{l} \aleph_{\phi} \sum_{k=0}^{m} f\left(y, \hbar_{u}, \hbar_{\theta}, \hbar_{\phi}\right), \sum_{k=0}^{m} \theta\left(y, \hbar_{u}, \hbar_{\theta}, \hbar_{\phi}\right), \sum_{k=0}^{m}\left(\eta, \hbar_{\theta}, \hbar_{\phi}\right) \mathrm{d} \eta
$$

According to Abolbashar et al. [57]

$$
\varphi_{m}^{t}=\varphi_{m}^{u}+\varphi_{m}^{\theta}+\varphi_{m}^{\phi}
$$

where $\varphi_{m}^{t}$ is the total square residual error. $\varphi_{m}^{t}$ is reduced to achieve the full value of convergence control parameters Minimize the values of convergence control parameters in MATHEMATICA 11.3 to achieve optimum values, $\hbar_{f}, \hbar_{\theta}$ and $\hbar_{\phi}$. Average square residual errors for $f(\eta), \theta(\eta)$ and $\phi(\eta)$ are computed respectively using the following formulae [51]

$$
\begin{aligned}
\varepsilon_{f} & =\frac{1}{j+1} \sum_{k=1}^{j}\left[A_{k}\left(e^{\eta} R_{f}^{2}(\eta)\right)_{\eta=x_{k}}\right], \\
\varepsilon_{\theta} & =\frac{1}{j+1} \sum_{k=1}^{j}\left[A_{k}\left(e^{\eta} R_{\theta}^{2}(\eta)\right)_{\eta=x_{k}}\right], \\
\varepsilon_{\phi} & =\frac{1}{j+1} \sum_{k=1}^{j}\left[A_{k}\left(e^{\eta} R_{\phi}^{2}(\eta)\right)_{\eta=x_{k}}\right]
\end{aligned}
$$

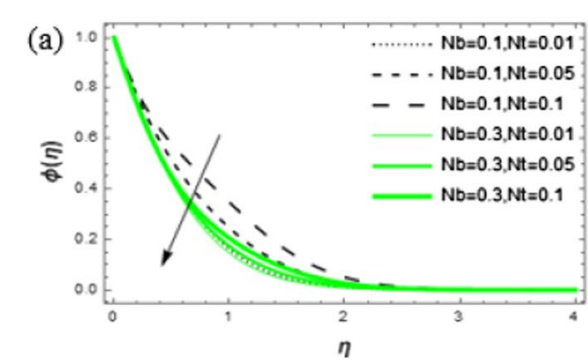

(c)

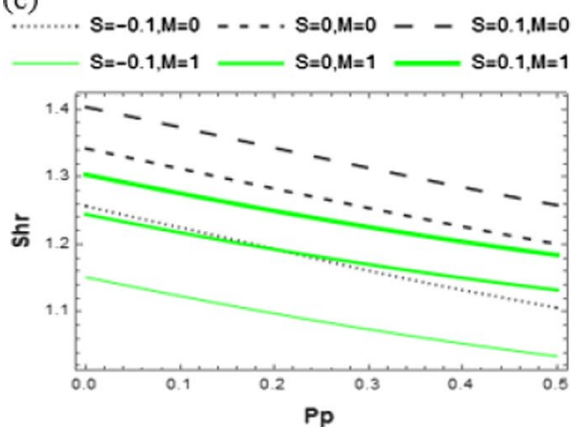

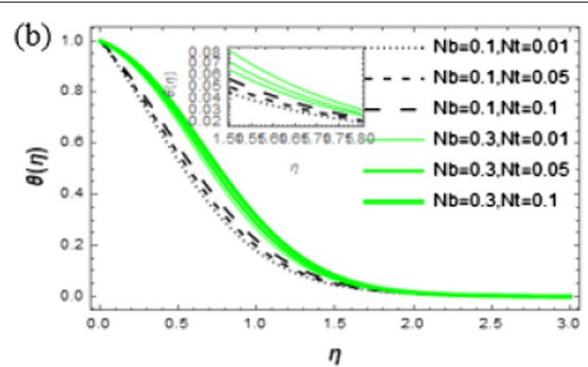

(d)
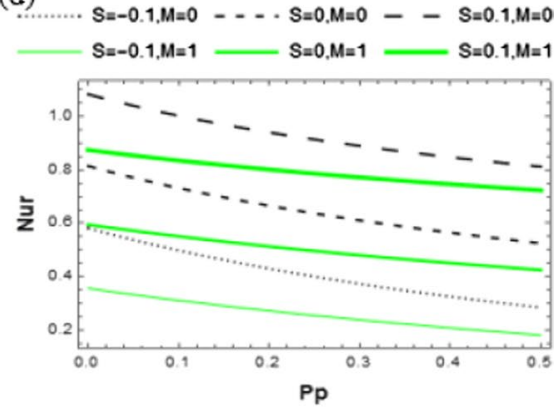

Fig. 7 a, b, c, and $\mathbf{d}$ Eckert number, chemical reaction, activation energy parameters, Brownian parameter, and thermophoresis parameter, suction parameter on fluid temperature, concentration profiles, Nusselt number, and Sherwood number 


\section{Validation, computational results, and discussion}

To get a clear understanding of the non-linear general model established, In this section, the numerical and analytical solutions of velocity, temperature, concentration, skin friction coefficient, Nusselt number, and Sherwood number are presented. Moreover, the effect of physical parameters on the profiles of velocity, temperature, and concentration is shown. MATHEMATICA software was used to carry out the program for the ordinary differential equations Equation.

Table 1 displays the convergence of CCM for the different orders of approximation. From Table 2 it was observed as the order of approximation increases, the optimum values of auxiliary parameters and the minimum values of square residual error are increased. Table 3 depicts the individual square residual error and convergence of the OHAM solution at various approximation orders. The OHAM solution is validated using the Chebyshev collocation method (CCM), and there is a strong match between the two results. To determine the accuracy of our method, a decent understanding was observed with previous published result Table 4. Table 5 shows the effect of $M, P p, \beta, E c$, $N b$, and $N t$. However, it is noticed that the values of Nusselt number elevate with $M$ $=P p, E c, \beta$, Whereas it reduces with increasing $N t$ and $N b$, also it is found that Sherwood number enhance $M, E c, N b$ Whereas it decreases with $P p$ and $N t$.

The direction skin friction coefficient increases as the Prandtl-number grows. While the degree of heat transfer rate additionally expands the convection heat and supply more to the fluid movement inside the boundary layer. It found that the magnitude of the skin friction coefficient had slightly decreased and the degree of heat transfer expansion in magnetic field force. Logically, the magnetic field force delivers an electromagnetic power that diminishes the level. However, direction skin friction coefficient heat transfer rate is more pronounced for Casson fluid compared with (Prandtl-number $=0.71$
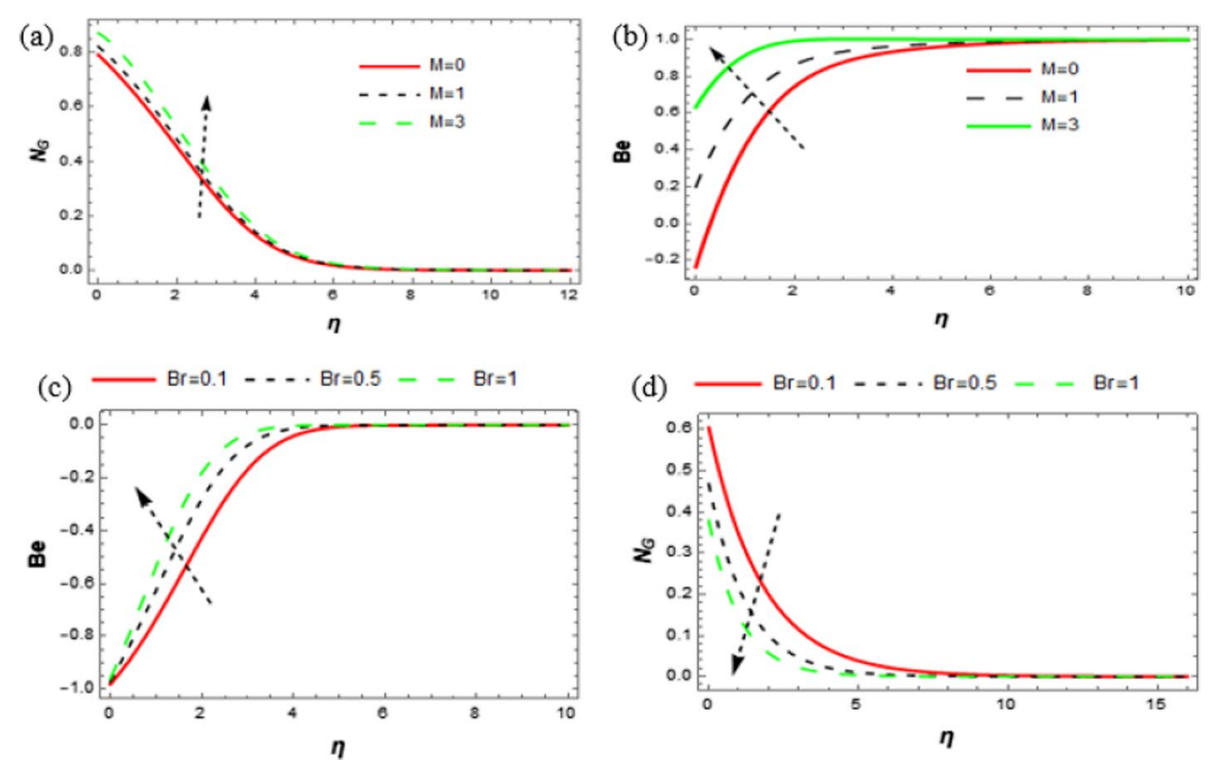

Fig. 8 a, b, c, and $\mathbf{d}$ Effect of magnetic field parameter (M), Brinkman number, on entropy generation, and Bejan number profile 
air) and (Prandtl-number $=7$ water). An increasing Eckert number decreases the direction of the skin friction coefficient. On the contrary, the higher value of the $E_{c}$ number improves the heat transfer rate. In the situation of (Prandtl-number $=0.71$ air) and (Prandtl-number $=7$ water), As the temperature increases, the skin friction coefficient and heat transfer rate drop in the fs and Pp, this observation is displayed in Tables 6 and 7.

To see the efficiency of the method used, simulation is given. The graphical effects of dimensionless velocity, temperature, and volume fraction of nanoparticles are compared (see Figs. 3, 4, 5) obtained using the Chebyshev collocation method (CCM) and Homotopy analysis method (OHAM). In each of the instances, there is a high degree of agreement.

Figure 6a depicts the effect of Casson and Magnetic field parameters on the velocity profile. The outcome indicates that the fluid velocity decreases with the Casson parameter. The Casson rheological parameter of acts as a fluid with a strong reaction to yield stress via plastic dynamic viscosity. It has also been found that the Casson rheological parameter decreases the reaction of non-Newtonian fluid. This recommends that the pure fluid act as a Newtonian fluid. However, the rheological velocity flow of the Casson boundary plate viscosity is greater when associated with the Newtonian fluid on contrary, the fluid temperature increases with Casson rheological parameter (see Fig. 6b). Figure 6c shows that as the magnetic field parameter increases, the fluid flow slows down. The existence of the Lorentz force can be attributed to that, which acts as a resistance force against fluid flow. Therefore, it slows the fluid speed while it can suppress and decelerate the movement of CF. From Fig. 6d the fluid temperature within the boundary layer speeds up due to increasing magnetic field parameters. Since ohmic heating serves as a supplementary heat source to the fluid temperature. Figure $6 \mathrm{c}, \mathrm{d}$ exhibit the impacts of porosity and porosity and firstorder slip parameters ( $\mathrm{Pp}$ and $\delta_{1}$ ) on fluid velocity. It was found that both porosity and first-order slip parameters. These results are perhaps due to the porosity parameter being inversely proportional to fluid permeability $\left(k_{p}\right)$. A rise in porosity parameter, manifests in increased resistance of the Casson nanofluid flow which leads to flow retardation. However, the first-order slip parameter decreases the velocity profile Subsequently, since not all the pulling force can be transmitted to the Casson nanofluid from the stretching sheet. This causes the fluid velocity to reduce. Eckert number has a significant effect on the temperature profile. This depicted that the physical case where more thermal energy is supplied as the Eckert number increase to boost the fluid temperature [8]. This allows the heat to conduct from the plate. This is because the thermal boundary layer is increased due to the conversion of kinetic energy into heat energy in the boundary layer with a greater viscous heating effect. From Fig. 7b It was found that the impacts of the chemical reaction and activation energy parameters on the nanoparticle volume fraction profile. The definition is obvious from Eq. (4) that the term $e^{\frac{-E}{(T r-1) \theta+1}} S c \sigma^{* 2}((\operatorname{Tr}-1) \theta+1)^{m}$ increases as $\sigma^{*}$ raised. This corresponds change the profile of the nanoparticle volume fraction. Besides, as the activation energy parameter $(E)$ is increased, the nanoparticle volume fraction profile is raised. The activation energy is the sum of energy that must be resolved before a chemical reaction can occur. An appreciable number of molecules with energy is significantly 
larger than the activation energy, which must be active to have a reasonable chemical reaction [62]. A rise in the activation energy parameter $(E)$ contributes to a decrease in the nanoparticle volume fraction profile of $S c \sigma^{* 2}((\operatorname{Tr}-1) \theta+1)^{m} e^{\frac{-E}{(T r-1) \theta+1}}$. As a function, the volume fraction profile of nanoparticles increases.

Figure $7 \mathrm{a}, \mathrm{d}$ reveals that the Increase in the Brownian motion parameter values triggers a rapid drop in the concentration profiles. The concentration profiles decrease with the reduction of the increases $\mathrm{Nb}$ parameter to a certain amount near the extending layer wall. The thermophoresis and Brownian motion both constitute two important processes of nanofluid flow. It is interesting to note that Brownian motion favours the growth of concentration level whereas thermophoresis reduces it. The concentration boundary layer near the wall decreases as $N b$ grows, but away from the wall. From this figure, it is seen that $N t$ is increasing to a certain value of $\eta$ near the stretching wall, but the opposite is beyond that. The pattern has been observed. This implies that the concentration boundary layer exists. Boundary Thickness grows, but only to a point, this is because of the altered nanoparticle concentration. Figure 7c displays the impact of suction parameters on the Nusselt number. Nusselt number decreases as a suction parameter $(S<0)$, decreases, but increases with suction parameter $(S>0)$. Also, Fig. 7d displays the impact of suction parameters on Sherwood number. The Nusselt number is observed to reduce as the suction parameter $(S<0)$. increases, whereas it rises as the injection parameter reduces.

Figure 8a-d Show the effect of Magnetic field parameter $(M)$, Brinkman Number, on entropy generation, and Bejan number profile. It was found that increasing the Brinkman number $(\mathrm{Be})$ contributes to convective heating and joule heating respectively, however, their effects generate an instability system. The situation of concentration irreversibility becomes more as the magnetic field parameter $(M)$ increases; the effect controls the operation factor in the entropy production. Consequently, the entropy generation rise in Fig. 8a while the opposite effect is observed for Fig. 8c. It should be noted that the Bejan number has a significant quantity ratio to produce entropy and also indicates the convective heat transfer ratio for the production of entropy. In general, the number of Bejan reveals numerical values ranging from 0 to 1 , where the number of Bejan is close to 1 , it shows that the production of the entropy is linked with the heat transfer that dominates. The Bejan number has therefore been increased by Fig. 8a, b.

\section{Conclusion}

Due to heat and mass transfer of Arrhenius activation energy and binary chemical reaction, the work has addressed the second-order velocity slip effect on reactive Casson nanofluid flow in a non-Darcian porous medium. Using CCS and OHAM, dimensionless conservation equations have been obtained and observed against various thermophysical parameters. The key findings of the present study are listed as:

1. For all physical parameter values used in this problem, both the OHAM and CCM provide a series of convergent solutions. 
2. The mounting values of Casson parameter, Magnetic field parameter, second-order slip, and wall transpiration (suction/injection) Eckert number, Brownian Parameter, and thermophoresis parameter have a significant effect on the temperature profile.

3. Chemical reaction, Arrhenius activation energy, Brownian Parameter, and thermophoresis parameter reduce the concentration field.

4. The effect of the Casson parameter, the magnetic field parameter reduces the velocity profile.

5. Both Nusselt number and Sherwood number profile become elevated for incrementing suction parameter $(S>0)$ while it is a detraction function of the injection parameter $(S<0)$.

6. The effect of Brinkman number and magnetic field parameter $(M)$ increases Bejan number profile contrary effect is found entropy production.

In the presence of Chemical reaction, Arrhenius activation energy, the presented analysis could be extended to explore the case of unsteady Casson nanofluids flow in a porous medium with the inference of solar radiation, Therefore Both OHAM and CCM, implementation can be expanded to include different and multi-dimensional flow problems.

\begin{abstract}
List of symbols
$u, v$ : Velocity in $x$ and $y$ directions; $\mu_{\infty}$ : Constant viscosity; $\rho_{\infty}$ : The density of the base fluid; $\beta$ : Casson parameter; $k(T)$ : Variable thermal conductivity; $\mu(T)$ : Variable viscosity; $D_{B}(C)$ : Variable mass diffusivity; $D T$ : Thermophoretic diffusion coefficient; $c_{p}$ : Specific heat at constant pressure; $k_{\infty}$ : Constant nanofluid thermal conductivity; $c_{s}$ : Specific heat of the solid surface; $J_{0}$ : Current density; $\nu_{\infty}$ : Constant kinematic viscosity; $\lambda$ : Latent heat of diffusion; $\tau$ : The ratio of nanoparticle heat capacity to the fluid heat capacity; $\left(k_{p}\right)$ : Permeability coefficient; $D_{B} \infty$ : Constant Brownian diffusion coefficient; $\bar{u}_{W}=\frac{U_{E \bar{T}}}{L}$ :The velocity of the sheet; $\lambda>0$ : Stretching sheet; $\bar{V}_{w}:$ Suction/injection; $\bar{u}_{\text {slip }}$ Velocity slip; $T_{\infty}$ : Ambient temperature; $T$ : Nanofluid temperature; $C_{w}$ : Wall nanoparticle volume fraction; $C_{\infty}$ : The ambient nanoparticle volume fraction; $a$ : Positive constant; $M$ : Magnetization in magnets; $s$ : Width of magnets and electrodes; $\alpha_{\infty}$ : Constant thermal diffusivity; $C$ : Nanoparticle volume fraction; E: Activation energy; $(S>0) /(S<0)$ : Suction/injection parameter.
\end{abstract}

\title{
Acknowledgements
}

The author appreciates the excellent research facilities provided by Kwara state university.

Authors' contributions

I am the individual author of the manuscript.

Funding

There is no funding for the research reported.

Availability of data and materials

All the data generated and materials during this study are included in this research article.

\section{Declarations}

\section{Competing interests}

The author declare that they have no competing interests.

\section{Author details}

${ }^{1}$ Department of Physics, Augustine University llara Epe, Lagos, Nigeria. ${ }^{2}$ Department of Statistics and Mathematical Sciences, Kwara State University, Malete, Nigeria.

Received: 16 March 2021 Accepted: 31 January 2022

Published online: 14 February 2022

\section{References}

1. Obalalu, A.M., Ajala, O.A., Abdulraheem, A., Akindele, A.O.: The influence of variable electrical conductivity on nonDarcian Casson nanofluid flow with first and second-order slip conditions. Part. Differ. Equ. Appl. Math. 4, 100084 (2021) 
2. Obalalu, A.M.: Heat and mass transfer in an unsteady squeezed Casson fluid flow with novel thermophysical properties: analytical and numerical solution. Heat Transf. 24, 1 (2021). https://doi.org/10.1002/htj.22263

3. Arrhenius, S.: Über die Änderung der Stärke schwacher Säuren durch Salzzusatz. J Zeitschrift für Physikalische Chemie. 31(1), 197-229 (1899). https://doi.org/10.1515/zpch-1899-3120

4. Li, W., Huang, J., Zhang, Z., Huang, H., Liang, J.: Evaluation and design methods for high-efficiency charring composite under complex coupling mechanisms in both material and boundary layer. J. Appl. Polym. Sci. 138(1), 49615 (2021). https://doi.org/10.1002/app.49615

5. Li, J., Tsona, N.T., Tang, S., Zhang, X., Du, L.: Influence of water on the gas-phase reaction of dimethyl sulfide with BrO in the marine boundary layer. ACS Omega (2021). https://doi.org/10.1021/acsomega.0c05945

6. Gonzalez Olivardia, F.G., Matsuo, T., Shimadera, H., Kondo, A.: Impacts of the tree canopy and chemical reactions on the dispersion of reactive pollutants in street canyons. J. Atmos. 12(1), 34 (2021). https://doi.org/10.3390/atmos 12010034

7. Obalalu, A.M., Wahaab, F.A., Adebayo, L.L.: Heat transfer in an unsteady vertical porous channel with injection/suction in the presence of heat generation. J. Taibah Univ. Sci. 14(1), 541-548 (2020). https://doi.org/10.1080/16583655. 2020.1748844

8. Bejan, A.: Entropy generation minimization: the new thermodynamics of finite-size devices and finite-time processes. J. Appl. Phys. 79(3), 1191-1218 (1996). https://doi.org/10.1063/1.362674

9. Kataria, H.R., Patel, H.R.: Radiation and chemical reaction effects on MHD Casson fluid flow past an oscillating vertical plate embedded in porous medium. Alex. Eng. J. 55(1), 583-595 (2016)

10. Obalalu, A.M., Ajala, O.A., Adeosun, A.T., Wahaab, F.A., Oluwaseyi, A., Adebayo, L.L.: Natural convective non-Newtonian Casson fluid flow in a porous medium with slip and temperature jump boundary conditions. Pet. Coal 62(4), 1532-1545 (2020)

11. Izadi, M., Srivastava, H.M.: A novel matrix technique for multi-order pantograph differential equations of fractional order. Proc. Roy. Soc. A 477(2253), 20210321 (2021)

12. Srivastava, H.M., Alomari, A.-K.N., Saad, K.M., Hamanah, W.M.: Some dynamical models involving fractional-order derivatives with the Mittag-Leffler type Kernels and their applications based upon the legendre spectral collocation method. Fractal Fractional. 5(3), 131 (2021)

13. Izadi, M., Srivastava, H.M.: A discretization approach for the nonlinear fractional logistic equation. Entropy $22(11)$, $1328(2020)$

14. Izadi, M., Srivastava, H.M.: Numerical approximations to the nonlinear fractional-order Logistic population model with fractional-order Bessel and Legendre bases. Chaos Solitons Fractals 145, 110779 (2021)

15. Hartmann, J., Lazarus, F.: Hg-dynamics II. Theory Laminar Flow Electric Conduct. Liq. Homogeneous Magn. Field 15, $7(1937)$

16. Wahaab, F.A., Adebayo, L.L., Adekoya, A.A., Hakeem, I.G., Alqasem, B., Obalalu, A.M.: Physiochemical properties and electromagnetic wave absorption performance of $\mathrm{Ni0} .5 \mathrm{Cu} 0.5 \mathrm{Fe}_{2} \mathrm{O}_{4}$ nanoparticles at $\mathrm{X}$-band frequency. J. Alloys Compd. 836, 155272 (2020). https://doi.org/10.1016/j.jallcom.2020.155272

17. Wahaab, F.A., Adebayo, L.L., Adekoya, A.A., Yusuf, J.Y., Obalalu, A.M., Yusuff, A.O., Alqasem, B.: Electromagnetic waveinduced nanofluid-oil interfacial tension reduction for enhanced oil recovery. J. Mol. Liq. 318, 114378 (2020). https:// doi.org/10.1016/j.molliq.2020.114378

18. Dawar, A., Shah, Z., Tassaddiq, A., Islam, S., Kumam, P.: Joule heating in magnetohydrodynamic micropolar boundary layer flow past a stretching sheet with chemical reaction and microstructural slip. Case Stud. Therm. Eng. (2021). https://doi.org/10.1016/j.csite.2021.100870

19. Ali, B., Naqvi, R.A., Mariam, A., Ali, L., Aldossary, O.M.: Finite element study for magnetohydrodynamic (MHD) tangent hyperbolic nanofluid flow over a faster/slower stretching wedge with activation energy. Mathematics $\mathbf{9}(1), 25$ (2021). https://doi.org/10.3390/math9010025

20. Noor, N.A.M., Shafie, S., Admon, M.A.: Impacts of chemical reaction on squeeze flow of MHD Jeffrey fluid in horizontal porous channel with slip condition. Phys. Scr. 96(3), 035216 (2021)

21. Wahaab, F.A., Adebayo, L.L., Rostami, A., Ganeson, M., Yusuf, J.Y., Afeez, Y., Obalalu, A.M., Abdulraheem, A., Oladosu, T.L.: Microwave absorption performance of $\mathrm{Ni0} .5 \mathrm{Zn0} 0.5 \mathrm{Fe}_{2} \mathrm{O}_{4}$ nanoclusters at $82-18 \mathrm{GHz}$ frequency. Indian J. Phys. (2021). https://doi.org/10.1007/s12648-021-02005-4

22. Naji, A., Azadkhah, S., Farahani, H., Uddin, S., Khan, F.R.: Microplastics in wastewater outlets of Bandar Abbas city (Iran): a potential point source of microplastics into the Persian Gulf. J. Chemosphere 262, 128039 (2021). https:// doi.org/10.1016/j.chemosphere.2020.128039

23. Yusuff, A.O., Yahya, N., Zakariya, M.A., Sikiru, S.: Investigations of graphene impact on oil mobility and physicochemical interaction with sandstone surface. J. Petrol. Sci. 198, 108250 (2021). https://doi.org/10.1016/j.molliq.2020. 114378

24. Yu,W., Xie, H.: A review on nanofluids: preparation, stability mechanisms, and applications. J. Nanomater. (2012). https://doi.org/10.1155/2012/435873

25. Gopal, D., Saleem, S., Jagadha, S., Ahmad, F., Almatroud, A.O., Kishan, N.: Numerical analysis of higher order chemical reaction on electrically MHD nanofluid under influence of viscous dissipation. Alexandria Eng. J. 60(1), 1861-1871 (2021). https://doi.org/10.1016/j.aej.2020.11.034

26. Obalalu, A.M., Adeosun, A.T., Wahaab, F.A., Aliu, O., Adebayo, L.L.: Natural convective non-Newtonian Casson fluid flow in a porous medium with slip and temperature Jump boundary. Pet Coal 62, 4 (2020)

27. Ramzan, M., Shaheen, N., Chung, J.D., Kadry, S., Chu, Y.-M., Howari, F.: Impact of Newtonian heating and Fourier and Fick's laws on a magnetohydrodynamic dusty Casson nanofluid flow with variable heat source/sink over a stretching cylinder. Sci. Rep. 11(1), 1-19 (2021). https://doi.org/10.1038/s41598-021-81747-x

28. Casson, N.: A flow equation for pigment-oil suspensions of the printing ink type. In: Rheology of Disperse Systems (1959)

29. Oyelakin, I.S., Mondal, S., Sibanda, P.: Unsteady Casson nanofluid flow over a stretching sheet with thermal radiation, convective and slip boundary conditions. Alexandria Eng. J. 55(2), 1025-1035 (2016). https://doi.org/10.1016/j.aej. 2016.03.003 
30. Mukhopadhyay, S., Vajravelu, K., Van Gorder, R.A.: Casson fluid flow and heat transfer at an exponentially stretching permeable surface. J. Appl. Mech.. 80, 5 (2013). https://doi.org/10.1115/1.4023618

31. Gad-El-Hak, M.: Gas and liquid transport at the microscale. Heat Transf. Eng. 27(4), 13-29 (2006). https://doi.org/10 1080/01457630500522305

32. Beskok, A., Karniadakis, G.E., Trimmer, W.: Rarefaction and compressibility effects in gas microflows (1996). https:// doi.org/10.1115/1.2817779

33. He, J.-H.: A short review on analytical methods for a fully fourth-order nonlinear integral boundary value problem with fractal derivatives. Int. J. Numer. Methods Heat (2020). https://doi.org/10.1108/HFF-01-2020-0060

34. Agarwal, P., Baltaeva, U., Alikulov, Y.: Solvability of the boundary-value problem for a linear loaded integro-differential equation in an infinite three-dimensional domain. Chaos Solitons Fractals 140, 110108 (2020). https://doi.org/10. 1016/j.chaos.2020.110108

35. Kudenatti, R.B., Bharathi, M.J.E.W.C.: Stability of hydromagnetic boundary layer flow of non-Newtonian power-law fluid flow over a moving wedge. Eng. Comput. (2020). https://doi.org/10.1007/s00366-020-01094-9

36. Mustafa, I., Abbas, Z., Arif, A., Javed, T., Ghaffari, A.: Stability analysis for multiple solutions of boundary layer flow towards a shrinking sheet: analytical solution by using least square method. Phys. A Stat. Mech. Appl. 540, 123028 (2020). https://doi.org/10.1016/j.physa.2019.123028

37. Obalalu, A.M., Kazeem, I., Abdulrazaq, A., Ajala, O.A., Oluwaseyi, A., Adeosun, A.T., Adebayo, L.L., Wahaab, F.A.: Numerical simulation of entropy generation for casson fluid flow through permeable walls and convective heating with thermal radiation effect. J. Serb. Soc. Comput. Mech. 14(2), 503-519 (2020). https://doi.org/10.24874/jsscm.2020.14. 02.10

38. Fazio, R., Jannelli, A.: A non-standard finite difference scheme for magneto-hydro dynamics boundary layer flows of an incompressible fluid past a flat plate. Math. Comput. Appl. (2021). https://doi.org/10.20944/preprints202102. 0125.v1

39. Goud, B.S., Kumar, P.P., Malga, B.S.: Effect of Heat source on an unsteady MHD free convection flow of Casson fluid past a vertical oscillating plate in porous medium using finite element analysis. Part. Differ. Equ. Appl. Math. 2, 100015 (2020). https://doi.org/10.1016/j.padiff.2020.100015Get

40. Mahdy, A., Chamkha, A.J., Nabwey, H.A.: Entropy analysis and unsteady MHD mixed convection stagnation-point flow of Casson nanofluid around a rotating sphere. Alex. Eng. J. 59(3), 1693-1703 (2020). https://doi.org/10.1016/j. aej.2020.04.028

41. Liao, S.: On the homotopy analysis method for nonlinear problems. Appl. Math. Comput. 147(2), 499-513 (2004). https://doi.org/10.1016/S0096-3003(02)00790-7

42. Ismail, A.: On new modifications of some perturbation procedures. Discrete Dyn. Nat. Soc. (2021). https://doi.org/10. $1155 / 2021 / 6681932$

43. Khan, Z.H., Gul, R., Khan, W.A.: Effect of variable thermal conductivity on heat transfer from a hollow sphere with heat generation using homotopy perturbation method. Heat Trans. Summer Conf. 48470, 301-309 (2008). https://doi. org/10.1115/HT2008-56448

44. Srivastava, H.M., Deniz, S., Saad, K.M.: An efficient semi-analytical method for solving the generalized regularized long wave equations with a new fractional derivative operator. J. King Saud Univ. Sci. 33(2), 101345 (2021)

45. Srivastava, H.M., Abdel-Gawad, H., Saad, K.M.: Oscillatory states and patterns formation in a model subjected to the Dirichlet conditions. Discrete Dyn. Syst. Ser. 14(10), 3785-3801 (2021)

46. Singh, H., Wazwaz, A.-M.: Computational method for reaction diffusion-model arising in a spherical catalyst. Int. J. Appl. Comput. Math. 7(3), 1-11 (2021)

47. Singh, H., Pandey, R.K., Singh, J., Tripathi, M.: A reliable numerical algorithm for fractional advection-dispersion equation arising in contaminant transport through porous media. Phys. A Stat. Mech. Appl. 527, 121077 (2019)

48. Singh, H.: Jacobi collocation method for the fractional advection-dispersion equation arising in porous media. Numer. Methods Part. Differ. Equ. (2020)

49. Singh, H.: Chebyshev spectral method for solving a class of local and nonlocal elliptic boundary value problems. Int. J. Nonlinear Sci. Numer. Simul. (2021)

50. Srivastava, H.M.: An introductory overview of fractional-calculus operators based upon the Fox-Wright and related higher transcendental functions. J. Adv. Eng. Comput. 5(3), 135-166 (2021)

51. Titiloye, E.O., Adeosun, A.T., Gbadeyan, J.A.: Influence of chemical reaction and arrhenius activation energy on hydromagnetic non-darcian casson nanofluid flow with second-order slip condition. Int. J. Eng. Res. Afr. 54, 100-117 (2021)

52. Parand, K., Hajimohammadi, Z.: Using modified generalized Laguerre functions, QLM and collocation method for solving an Eyring-Powell problem. J. Braz. Soc. Mech. Sci. Eng. 40(4), 1-9 (2018)

53. Ajala, O., Adegbite, P., Abimbade, S.F., Obalalu, A.M.: Thermal radiation and convective heating on hydromagnetic boundary layer flow of nanofluid past a permeable stretching Surface. Int. J. Appl. Math. Stat. Sci. 2(8), 3219-3972 (2019)

54. Ajala, O.A., Akinyemi, O.: Numerical study of forced convective heat generation flow through a permeable walls with Suction/injection. Int. J. Sci. Res. Publ. 9(6), 336-348 (2019)

55. Ajala, O., Adegbite, P., Abimbade, S., Obalalu, A.:Thermal radiation and convective heating on hydro magnetic boundary layer flow of nanofluid past a permeable stretching surface. Int. J. Appl. Math. Stat. Sci. (2019)

56. Ajala, O.A., Obalalu, A.M., Akinyemi, O.T., Abimbade, S.F.: Numerical study of forced convective heat generation flow through a permeable walls with suction/injection. Int. J. Sci. Res. Publ. 9, 6 (2019). https://doi.org/10.29322/IJSRP.9. 06.2019.p9094

57. Abolbashari, M.H., Freidoonimehr, N., Nazari, F., Rashidi, M.M.: Analytical modeling of entropy generation for Casson nano-fluid flow induced by a stretching surface. J. Adv. Powder Technol. 26(2), 542-552 (2015). https://doi.org/10. 1016/j.apt.2015.01.003

58. Srivastava, H.M.: Some parametric and argument variations of the operators of fractional calculus and related special functions and integral transformations. J. Nonlinear Convex Anal. 22, 1501-1520 (2021) 
59. Srivastava, H.M.: Fractional-order derivatives and integrals: Introductory overview and recent developments. Kyungpook Math. J. 60(1), 73-116 (2020)

60. Khan, W., Pop, I.: Boundary-layer flow of a nanofluid past a stretching sheet. Int. J. Heat 53(11-12), 2477-2483 (2010). https://doi.org/10.1016/j.ijheatmasstransfer.2010.01.032

61. Makinde, O.D., Aziz, A.: Boundary layer flow of a nanofluid past a stretching sheet with a convective boundary condition. Int. J. Therm. Sci. 50(7), 1326-1332 (2011). https://doi.org/10.1016/j.ijthermalsci.2011.02.019

62. Obalalu, A.M., Ajala, O.A., Adeosun, A.T., Akintayo, A.O., Oladapo, O.A., Akintayo, O.O., Peter, A.: Significance of variable electrical conductivity on non-Newtonian fluid flow between two vertical plates in the coexistence of Arrhenius energy and exothermic chemical reaction. Part. Differ. Equ. Appl. Math. 4, 100184 (2021)

\section{Publisher's Note}

Springer Nature remains neutral with regard to jurisdictional claims in published maps and institutional affiliations.

\section{Submit your manuscript to a SpringerOpen ${ }^{\circ}$} journal and benefit from:

- Convenient online submission

- Rigorous peer review

- Open access: articles freely available online

- High visibility within the field

- Retaining the copyright to your article

Submit your next manuscript at $\gg$ springeropen.com 